\title{
A color-period diagram for the open cluster M 48 (NGC 2548), and its rotational age $\mathrm{e}^{\star, \star \star, \star \star \star}$
}

\author{
Sydney A. Barnes ${ }^{1,2}$, Joerg Weingrill ${ }^{1}$, Thomas Granzer ${ }^{1}$, Federico Spada ${ }^{1}$, and Klaus G. Strassmeier ${ }^{1}$ \\ ${ }^{1}$ Leibniz Institute for Astrophysics Potsdam (AIP), An der Sternwarte 16, 14482 Potsdam, Germany \\ e-mail: [sbarnes; jweingrill; tgranzer;fspada;kstrassmeier]@aip.de \\ 2 Space Science Institute, 4750 Walnut Street, Boulder, CO 80301, USA \\ Received 18 March 2015 / Accepted 3 September 2015
}

\section{ABSTRACT}

\begin{abstract}
Rotation periods are increasingly being used to derive ages for cool single field stars. Such ages are based on an empirical understanding of how cool stars spin down, acquired by constructing color-period diagrams (CPDs) for a series of open clusters. Our main aims here are to construct a CPD for M 48, to compare this with other clusters of similar age to check for consistency, and to derive a rotational age for M48 using gyrochronology. We monitored M 48 photometrically for over 2 months with AIP's STELLA I $1.2 \mathrm{~m}$ telescope and the WiFSIP 4K imager in Tenerife. Light curves with 3 mmag precision for bright $(V \sim 14$ mag) stars were produced and then analysed to provide rotation periods. A cluster CPD has then been constructed. We report 62 rotation periods for cool stars in M 48. The CPD displays a clear slow/I-sequence of rotating stars, similar to those seen in the $625 \mathrm{Myr}$-old Hyades and $590 \mathrm{Myr}$-old Praesepe clusters, and below both, confirming that M 48 is younger. A similar comparison with the 250 Myr-old M 34 cluster shows that M 48 is older and does not possess any fast/C-sequence $\mathrm{G}$ or early $\mathrm{K}$ stars like those in M 34, although relatively fast rotators do seem to be present among the late-K and $\mathrm{M}$ stars. A more detailed comparison of the CPD with rotational evolution models shows that the cluster stars have a mean age of $450 \mathrm{Myr}$, and its (rotating) stars can be individually dated to $\pm 117 \mathrm{Myr}$ (26\%). Much of this uncertainty stems from intrinsic astrophysical spread in initial periods, and almost all stars are consistent with a single age of $450 \mathrm{Myr}$. The gyro-age of M 48 as a whole is $450 \pm 50 \mathrm{Myr}$, in agreement with the previously determined isochrone age of $400 \pm 100 \mathrm{Myr}$.
\end{abstract}

Key words. stars: activity - stars: rotation - stars: late-type - stars: evolution - open clusters and associations: individual: M 48 stars: variables: general

\section{Introduction}

The study of stellar rotation, both among field stars and in open clusters, was synonymous with $v$ sin $i$ measurements for several decades. See Kraft (1970), and references therein, for a review of developments until 1970. Good starting points for developments into the 1980s and 1990s, especially those relating to open clusters, are Stauffer \& Hartmann (1987), Soderblom et al. (1993), and Queloz et al. (1998). More recently the emphasis has shifted to photometric rotation period measurements, partly because they avoid the $\sin i$ ambiguity inherent to spectroscopic measurements.

Such measurements began with the pioneering work of Van Leeuwen et al. (1987) ${ }^{1}$, who measured rotation periods photometrically for $11 \mathrm{cool}$ stars in the Pleiades open cluster, and were soon followed by the remarkable Hyades rotationperiod measurements ${ }^{2}$ of Radick et al. (1987). The Mt. Wilson

* Based on data obtained with the STELLA robotic telescopes in Tenerife, an AIP facility jointly operated by AIP and IAC; this paper presents results for the STELLA Open Cluster Survey (SOCS).

$\star \star$ Appendices $\mathrm{A}$ and $\mathrm{B}$ are available in electronic form at http: //www . aanda. org

$\star \star \star$ The cluster photometry table is only available at the CDS via anonymous ftp to cdsarc.u-strasbg.fr (130.79.128.5) or via http://cdsarc.u-strasbg.fr/viz-bin/qcat?J/A+A/583/A73

1 See also Van Leeuwen \& Alphenaar (1982).

2 No older cluster was measured successfully until Meibom et al. (2011b) studied the 1 Gyr-old cluster NGC 6811 using the Kepler Space Telescope. sample of stars, with periods measured from variability in chromospheric emission (Baliunas et al. 1996) and rooted in earlier spectroscopic work, including the discovery of stellar chromospheric activity cycles (Wilson 1978), is also noteworthy.

A large body of subsequent work by the astronomical community (e.g., Bouvier et al. 1993 (T Tauri stars), Barnes et al. 1999 (IC 2602); Irwin et al. 2007 (NGC 2516); Meibom et al. 2009 (M 35); Hartman et al. 2010 (Pleiades)) has shown that rotation period measurements constitute a distinct new probe of stellar evolution that provides both similar and new information, as compared with "classical" methods, such as isochrone fitting of color-magnitude diagrams (CMDs), from which it is steadily becoming independent. Such independence is valuable because young clusters contain few (or sometimes even no) bona fide giant members, making isochrone fitting particularly challenging.

Indeed, it is now recognized that color-period diagrams (CPDs) of open clusters are similar to CMDs, and provide a useful complementary means of characterizing open clusters, particularly in terms of ranking them by age (e.g., Barnes 2003; Meibom et al. 2015). Making the reasonable assumption that stars in both open clusters and the field spin down in similar ways because spindown is governed by processes internal to stars allows field star ages and those of any accompanying planets to be derived using gyrochronology (e.g., Barnes 2007), a valuable ability in the Kepler (and soon PLATO) era.

Another notable aspect of open cluster CPDs is that they often display sequences of rotating stars (Barnes 2003). Clusters like the Hyades (Radick et al. 1987; Delorme et al. 2011) 
and NGC 6811 (Meibom et al. 2011b) display a clear slow or I-sequence (consisting of relatively slowly-rotating stars whose periods increase steadily as cool star spectral types change from F- to G- to K-type). These stars have converged onto this sequence over the several-100 Myr ages of these clusters. Zero-age main sequence clusters, such as IC 2391 (Patten \& Simon 1996) and IC 2602 (Barnes et al. 1999), display only weak evidence for such a sequence. (However, the paucity of available stars could play a role in the difficulty of recognizing it in such cases.)

Certain young clusters, such as M 35 (Meibom et al. 2009), and the Pleiades (Hartman et al. 2010), display evident fast/ $\mathrm{C}$-sequences of rotating stars (consisting of many G-, K-, and M-type stars with rotation periods, $P \lesssim 1 \mathrm{~d}$ ), in addition to the slow/I-sequence that characterizes older clusters. This fast sequence appears to dissipate rapidly on a timescale that depends on stellar mass, because these stars spin down to populate the cluster's slow sequence instead. This is still a subject of active research. For recent ideas, see Matt et al. (2015), Gallet \& Bouvier (2015), Brown (2014), Epstein \& Pinsonneault (2014), and references therein. Earlier ideas concerning slow- and fastrotating stars (with varying emphasis on the mass dependence of rotation suggested by rotational sequences) can be found in, e.g., MacGregor \& Brenner (1991), Chaboyer et al. (1995), Collier-Cameron et al. (1995), Barnes \& Sofia (1996), Bouvier et al. (1997), and Sills et al. (2000).

A significant part of the difficulty in understanding the morphological changes that occur between the Pleiades-type ( $\$ 200 \mathrm{Myr})$ clusters and the Hyades-type $(\sim 600 \mathrm{Myr})$ clusters is the lack of appropriate published rotation-period observations for clusters of intermediate age. The only one available to date is the M 37 cluster (Hartman et al. 2009), whose rotational age has been claimed to be younger than its $540 \mathrm{Myr}$ isochrone age. With an isochrone age of $400 \mathrm{Myr}$ (Balaguer-Nunez et al. 2005), M 48 lies squarely in this intermediate age range and could help in elucidating the transitional rotational behavior. It is therefore desirable to construct a reliable CPD to characterize the M48 cluster and to explore its properties empirically in the context of other well-studied clusters. In particular, a CPD would allow a comparison between the age determined from rotation and the age determined from classical isochrone fitting.

More generally, such studies also increase our basic knowledge (e.g., photometry and membership) of the often unknown lower main sequence populations of these clusters, a difficult task in the pre-CCD era. We have studied the open cluster M 48 (NGC 2548) in this context. An additional context is provided by the STELLA Open Cluster Survey, which aims to provide rotation periods for a series of open clusters. For a related study of the IC 4756 cluster, see Strassmeier et al. (2015).

Despite being a Messier (1781) object $^{3}$, M $48\left(\alpha_{2000}=\right.$ $081343, \delta_{2000}=-054500$ ) has not been the subject of many prior studies, a fact that may be related to its being located in the southern sky. Ebbighausen (1939) performed a proper motion study of the upper main sequence (B and A spectral types) and giants of the cluster within a $15^{\prime}$ radius, and identified 74 of these stars as probable members. Another dedicated cluster study was not published for more than 60 years, until $\mathrm{Wu}$ et al. (2002) performed another proper motion study of a $1.6 \times 1.6^{\circ}$ region around the cluster and identified 165 stars as probable cluster members. This particular paper did not provide any photometric information. However, a related study by Balaguer-Nunez et al. (2005; hereafter BJG05) has revealed that the Wu et al. (2002)

\footnotetext{
3 The cluster apparently lies $2.5^{\circ}$ south of Messier's position, and was identified with NGC 2548 by Oswalt Thomas in 1934.
}

astrometric cluster members were brighter than $V \approx 13$. BJG05 focused on providing multicolor Stromgren photometry for a cluster-centered $34^{\prime} \times 34^{\prime}$ region to a depth of $V \approx 22$, and using this photometry they constructed a candidate member list to a depth of $V=18$. They also (re-)determined the cluster's basic parameters, including the age, $400 \pm 100 \mathrm{Myr}$.

Almost contemporaneously, Rider et al. (2004) provided photometry in the Sloan filter system for the cluster region to a depth of $g_{0}^{\prime}=16$ and found that a $400 \mathrm{Myr}$ isochrone matched their photometry reasonably well. Finally, the most recent work on this cluster by Wu et al. (2006) provided 13-band photometry in a specialized (BATC) filter set over a $58^{\prime} \times 58^{\prime}$ field, and identified 323 stars as (SED-based) photometric candidate cluster members. An agreement level of $80 \%$ was claimed between this membership criterion and earlier proper motion studies. Curiously, no photometry in Johnson colors is currently available for this cluster beyond the bright stars studied photoelectrically by Pesch (1961) and the (V,I) study of Sharma et al. (2006), the latter restricted to stars within an $8^{\prime}$ radius of the cluster center. The study presented here builds on these prior ones with an emphasis on the rotational properties of the cluster's stars.

The rest of this paper is organized as follows. The observations are discussed in Sect. 2. We present the cluster colormagnitude diagram in Johnson $B$ and $V$ colors to a depth of $V \sim 20$ in Sect. 3. The variability analysis is presented in Sect. 4, leading to the construction of the cluster color-period diagram, followed by relevant comparisons. Finally, the conclusions are presented in Sect. 5.

\section{The observations}

The M 48 open cluster was observed with the WiFSIP 4K CCD imager mounted on the AIP's STELLA I robotic $1.2 \mathrm{~m}$ telescope, located at the IAC in Tenerife, Spain (Longitude: $16^{\circ} 30^{\prime} 35^{\prime \prime}$ West, Latitude: $\left.28^{\circ} 18^{\prime} 00^{\prime \prime}\right)$. The STELLA robotic observatory (also containing a $1.2 \mathrm{~m}$ spectroscopic telescope, STELLA II) opens and closes automatically every usable night, guided by measurements of a number of weather and meteorological parameters. During this interval, it observes a set of targets that are chosen by a scheduling program on the basis of user-defined priorities. Details about the facility and its operation may be found in Strassmeier et al. (2004), Granzer (2004), and Strassmeier et al. (2010).

The cluster was observed nightly (as allowed by weather) over a two-month baseline from 4 March 2014 to 7 May 2014. A $44^{\prime} \times 44^{\prime}$ field, consisting of a $2 \times 2$ mosaic of $22^{\prime}$ CCD fields (NW, NE, SW, SE) centered on the cluster, was monitored. Our field is significantly larger than the region covered by BJG05, as can be seen in Fig. 1. Having this large a field was fortuitous, because cluster stars and rotators extend across the entire region monitored. In fact, the cluster very likely extends significantly beyond even our study field ${ }^{4}$. Generally, the telescope cycled sequentially through the four subfields. The time-series exposures were acquired in the Johnson $V$ band with exposure times of $30 \mathrm{~s}$ (short) and $300 \mathrm{~s}$ (long). The number of visits (either exposure time) per night per field ranged from zero to 7 , as permitted by other scheduled programs, weather, and telescope performance.

Over the observing period, we acquired a total of 1481 shortand long-exposure frames for the four fields. Of these, we (conservatively) discarded 992 because of tracking errors, lunar

4 For instance, the rotators we have identified below extend to the edges of the observed field. 


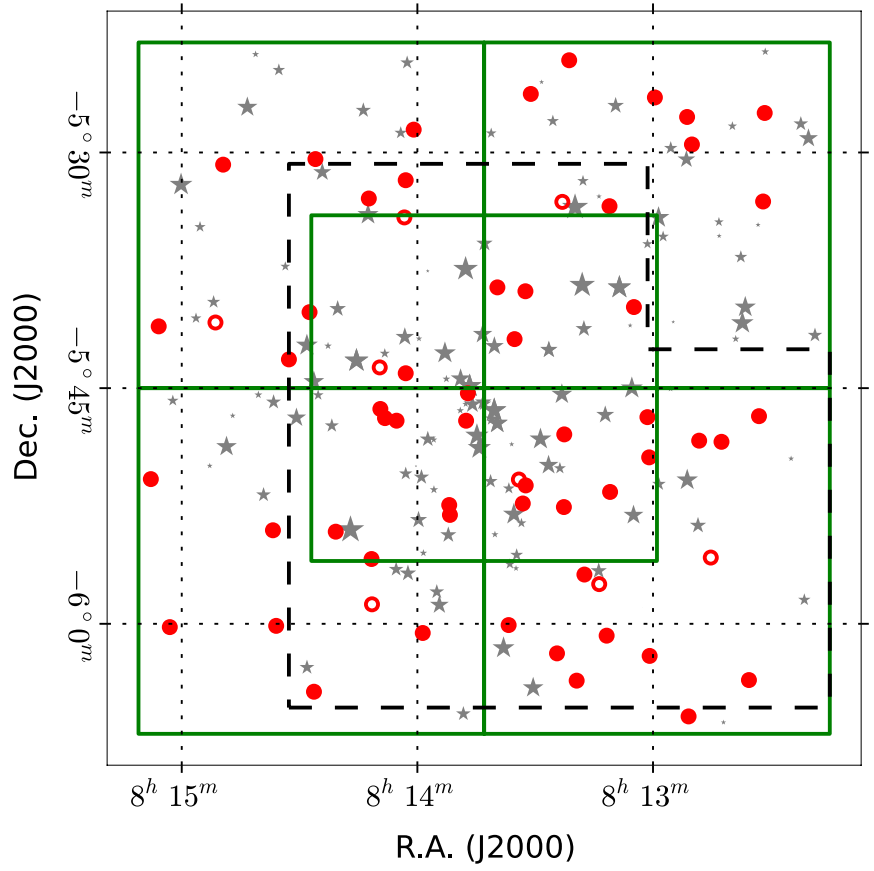

Fig. 1. On-sky areal coverage of our photometry (a $2 \times 2$ CCD mosaic covering a $44^{\prime} \times 44^{\prime}$ region and an additional central field) in the region of the M 48 cluster is displayed with solid gray lines (green online). The dashed lines indicate the (smaller) region covered by BJG05. Stars brighter than $V=12$ are marked, with symbol sizes scaled inversely with their $V$ magnitudes. The locations of the 62 identified rotating stars (discussed later) are also indicated with circular symbols.

proximity, bad seeing, or conditions deemed too far from photometric for our purposes, leaving us with 489 high-quality visits to the four cluster fields (average $=122 /$ field) for the time series observations. Small differences in the numbers of images for each sub-field arise from variations in observing conditions, and because our observing program did not modify individual field observing priorities after the acquisition of incomplete nightly observing cycles.

In addition, sets of Johnson $B$ and $V$ exposures were obtained for each of the cluster sub-fields on multiple nights, together with standard star observations of Landolt (2009) standard fields. This allowed us to obtain colors for the CPD, and of course, to construct a CMD for the cluster in Johnson colors. However, because the number of standard stars observed was small, we decided to place our final photometry on the photoelectric system of Pesch (1961). We also specifically acquired photometry for a separate field bore-sighted on the cluster center, to verify and ensure that the $2 \times 2$ mosaic of cluster sub-fields are placed on a common photometric system.

As part of the robotic observations, bias and flat field frames are obtained before and after science observations. Two bracketing master bias frames, each consisting of 25 individual frames, are used for bias subtraction on the science frames. No dark current subtraction is performed as the dark current is below $1 \mathrm{e}^{-} / \mathrm{h}$ at the nominal operation temperature. With the limited time available for twilight sky flats, it is impossible to flat field all of the 21 filters of WiFSIP on a single night. Instead, each twilight phase is used to calibrate 2-3 filters with 10 individual flat exposures each, grouped in two five-exposure sequences at opposite derotator settings to level-out first order illumination gradients remaining in twilight sky flats for imaging instruments with large fields of view (Chromey \& Hasselbacher 1996). The master flat field for individual science frames is then constructed
Table 1. Dates and numbers of secondary standard stars.

\begin{tabular}{lcc}
\hline \hline Field & Date & Number of stars \\
\hline M 48 BVI NE & 2014-02-28 & 184 \\
M 48 BVI NE & $2014-03-01$ & 184 \\
M 48 BVI NW & $2014-03-03$ & 192 \\
M 48 BVI C & $2014-03-06$ & 211 \\
M 48 BVI SE & $2014-03-05$ & 209 \\
M 48 BVI SE & $2014-03-02$ & 208 \\
M 48 BVI SW & $2014-03-07$ & 205 \\
\hline
\end{tabular}

by averaging at least ten such flat field blocks, but allowing for up to 100 blocks as long as a maximum time difference between flat block and science frame of less than \pm 25 days is not exceeded. The small variations in image scale across the field of view lead to each pixel receiving light from differing solid angles, in turn leading to differing light levels even on perfectly flat-illuminated fields. The average master flat field is corrected for this geometrical effect before usage on the science image.

For multi-amplifier readout modes like ours, crosstalk between the amplifiers can lead to ghost signals on corresponding pixel positions. Although it is a minor effect of a few tenths of a per cent, raw images are corrected for crosstalk. Subtle gain variations between the different amplifiers are compensated for by adjusting the amplifier gain to allow for a steady transition in illumination level across the amplifier read-out edges. Again, these variations are a few tenths of a per cent, compared to the nominal gain factors of 1.568 and 1.587 , for the two amplifiers used; however, the precision desirable for our time-series analysis makes such compensation advisable. Before performing the final photometry, the astrometric world-coordinate solution is obtained using a modified version of WCSTools (Mink 2002). The final solution follows the FITS conventions (Calabretta \& Greisen 2002) for a zenithal polynomial projection (ZPN) to third degree, with the remaining root-mean square (rms) error in the position on the order of 0.2 arcsec.

\subsection{Photometry}

Photometry for each frame was performed with SExtractor (Bertin \& Arnouts 1996) using the ISOCORR aperture. This choice was preferable to others such as FIXED APERTURE or ISOAUTO because it worked better than those choices in this work, principally because our point spread function (PSF) is sometimes distorted by tracking errors and/or less-than-perfect seeing conditions. Using an adaptive aperture preserves the most frames for photometry, with minimal impact on the photometric quality.

For each field, the frame with the highest ratio of matched to identified sources in the PPMXL catalog was selected as the reference frame. This selection was also verified manually and showed that only photometric nights were chosen. The reference frames for all five fields $(2 \times 2$ mosaic + central field $)$ ended up being selected from only a few photometric nights ( 28 Feb. 2014 to 7 Mar. 2014). The particular fields standardized on each night are listed in Table 1.

Up to five frames having the smallest offsets with respect to the reference frame for stars in the range between 10 to $16 \mathrm{mag}$ were then selected. These offsets calculated from the mean differences between the stars in the reference frame and the cross-identified ones in the selected frames were typically less than 4 millimag, with an rms below 1 millimag. The mean magnitude for each star was calculated from the five selected frames. 
The nightly offset of the reference frame to the Landolt standard fields was finally added to the derived magnitudes. Only stars within a $0.15^{\prime \prime}$ matching radius were identified as crossmatched. The whole procedure was performed separately for the $B$ and $V$ frames.

\section{Color-magnitude diagram}

$B$ and $V$ frames from the best photometric nights were manually selected for the CMD. For these frames the successive magnitude determinations for the best-exposed stars were repeatable at the 3 mmag level. We decided simply to place the frames on the (photoelectric) photometric standard system of Pesch (1961).

We matched 36 stars from Pesch (1961) listed on Simbad and Webda with our dataset. Webda lists 37 stars from Pesch and 14 stars from Oja (1976, priv. comm.). Since the Oja sample included fainter stars, and proved to be of similar photometric quality, we included them in our calibration sample. Four stars out of the initially 40 cross-identified ones were omitted for calibration due to large differences in $V$ magnitude or $B-V$ color (TYC 4859-28-1, HD 68779, BD-05 2451, BD-05 2452). This might arise from coordinate mismatching or bad photometry. Eventually 30 stars from Pesch and 6 stars from Oja were retained for calibration. None of our calibration stars showed saturated pixels in the PSF. The $V$ magnitude of the calibration stars covered a range from $8.184 \mathrm{mag}$ to $14.272 \mathrm{mag}$ and $0.001<$ $B-V<1.441$ in color. For these 36 stars we achieved an rms of $0.032 \mathrm{mag}$ in $V_{\text {STELLA }}-V_{\text {Pesch } / \text { Oja }}$ and $0.020 \mathrm{mag}$ in $(B-V)_{\text {STELLA }}-(B-V)_{\text {Pesch/Oja }}$.

The CMD in Johnson $B-V$ color for the entire $44^{\prime} \times 44^{\prime}$ survey area of our study is displayed in the upper panel of Fig. 2. The cluster's main sequence is obvious, and is reasonably distinguishable visually from the background stars in this direction of the Galaxy. The cluster sequence begins somewhat brighter than $V=10$, which is where the A-type cluster stars are located, and extends diagonally downward to $V \sim 19$, where the cluster's M-type stars become indistinguishable from the field population. A binary sequence, while undoubtedly present, is not visually prominent. A hint of a white dwarf sequence is visible. Our scientific interests center on the fainter $(V>13)$ cool FGK stars. Photometric information for all stars and cross-identifications with BJG05 are provided in an accompanying table available at the CDS.

It is desirable to understand which of these stars have been determined to be cluster members by prior studies, and to use this information where possible. The deepest of these studies is that by BJG05. Beginning with the Wu et al. (2006) astrometric members, which run out at a depth of $V \sim 13$, they extended the candidate member list downward to $V \sim 18$ using multi-color Stromgren photometry to select cluster members. We have crossidentified the cluster members selected by Balaguer-Nunez et al. (2005) against our photometry, and have marked these stars in the color-magnitude diagram displayed in the lower panel of Fig. 2. (As an aside, we note that BJG05 defined the cluster sequence using an empirical ZAMS constructed by Crawford 1975 and succeeding authors as referenced in BJG05.) We observe that the vast majority of the members identified by BJG05 indeed lie on the cluster's sequence in our $(B, V)$ photometry. It therefore appears that the BJG05 selection is an inclusive, rather than an exclusive one.

While we are fortunate that this prior membership information from BJG05 is available for a significant fraction of the stars of interest in our study, our study area is somewhat larger than theirs (see Fig. 1), so it will not be a surprise that we are
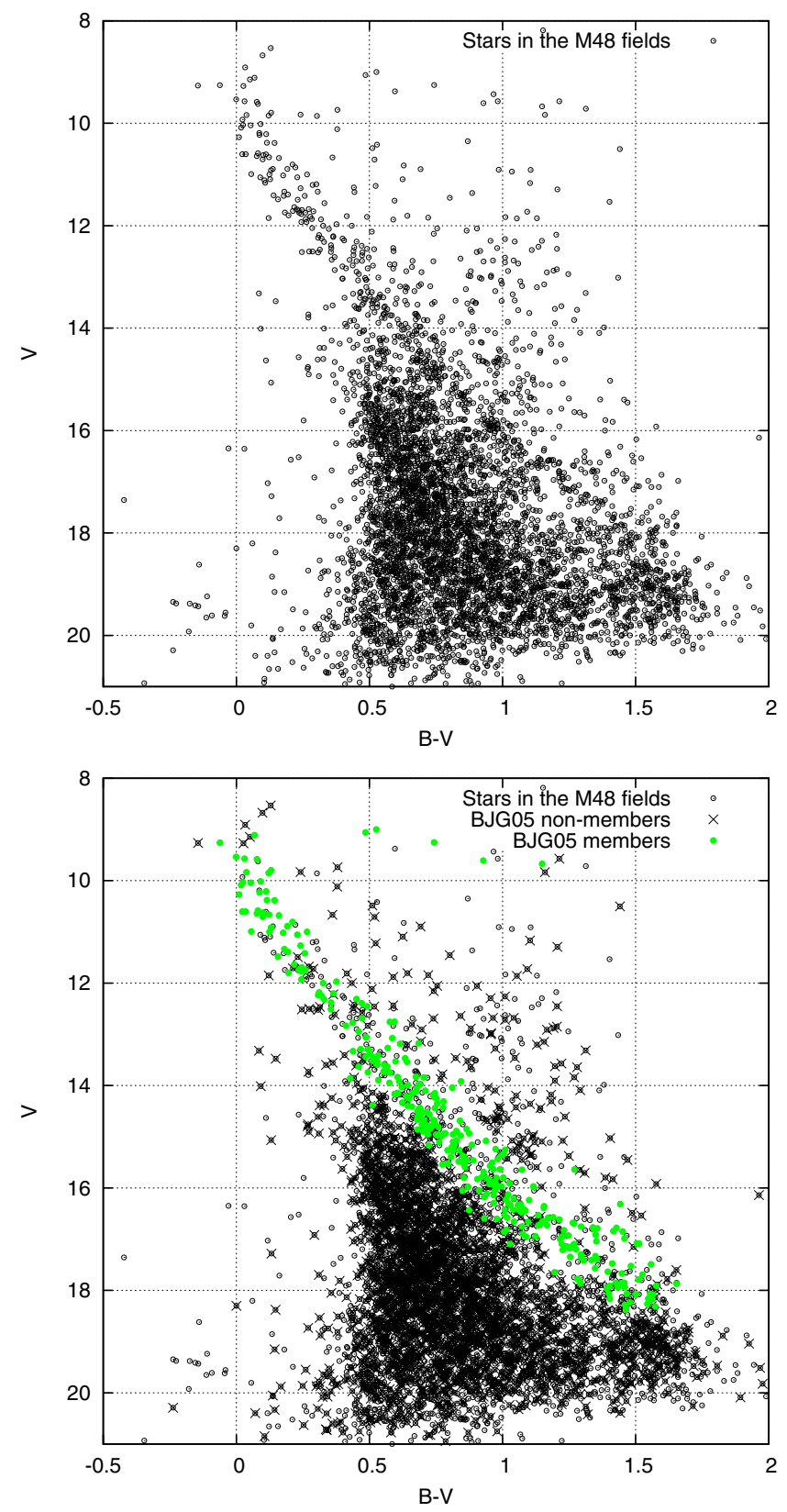

Fig. 2. Upper panel: color-magnitude diagram (CMD) for the $44^{\prime} \times 44^{\prime}$ M48 cluster region of our study in Johnson $B-V$ color. A cluster sequence, beginning brighter than $V=10$ and extending diagonally downward to $V \sim 19$, is seen clearly against the field population. Lower panel: large filled gray circles (green online) in the M48 CMD indicate cluster members identified by BJG05, based on astrometry brighter than $V=13$, and multi-color Stromgren photometry for fainter stars. Most of these BJG05 members are clearly on the cluster sequence in our photometry. Stars proposed by BJG05 to be non-members are additionally indicated with crosses, while the remaining empty circles have no BJG05 membership information.

able to propose additional candidate cluster members, some of them even with determined periods. Accordingly, a number of our rotational candidate cluster members (see Table A.1 below) are classified as "-", indicating that they do not have a BJG05 designation. However, these stars are both on the cluster's photometric sequence in our $(B, V)$ color-magnitude diagram, and have measured rotation periods consistent with cluster membership. (In Table A.1, M = BJG05 member, and N = BJG05 nonmember.) Ultimately, the BJG05 cluster member selection and 


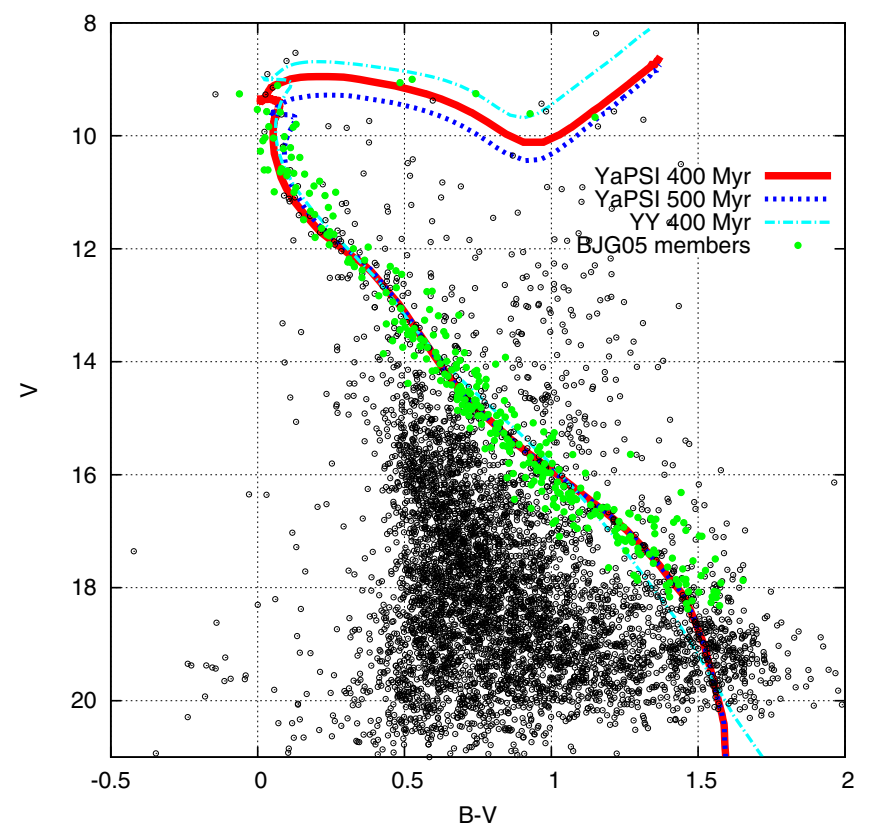

Fig. 3. CMD for M 48 with solar-metallicity isochrones based on Spada et al. (2013; YaPSI) for ages of 400-, and $500 \mathrm{Myr}$, and from Yi et al. (2001; YY) for $400 \mathrm{Myr}$. The distance modulus and reddening used are $(m-M)=9.3$ and $E(B-V)=0.08$ respectively.

ours are both photometric below $V=13$, the primary region of interest for this work, and therefore neither can be considered definitive in this region.

The relative absence of giant cluster members makes the photometry sub-optimal for an isochrone fit. However, it is certainly useful to compare the observed cluster main sequence with a modern theoretical isochrone. Accordingly, we have calculated (and display in Fig. 3) three suitable isochrones, based on the models of Spada et al. (2013) and of Yi et al. (2001).

One of the goals of Spada et al. (2013) was to update the Yale-Yonsei $\left(Y^{2}\right)$ database of stellar models (Yi et al. 2001), with particular attention to the input physics relevant for the lower mass regime (i.e., $\left.M \lesssim 0.6 M_{\odot} ;(B-V)_{0} \gtrsim 1.3\right)$. Most notably, the atmospheric boundary conditions are based on the PHOENIX model atmospheres (Hauschildt et al. 1999; Allard et al. 2011). For more details on the micro-physics used in the models, see Spada et al. (2013).

The isochrones have been calculated for solar metallicity, with ages of $400 \mathrm{Myr}$ (the nominal cluster age), and $500 \mathrm{Myr}$. These are displayed in Fig. 3, assuming a distance modulus of $9.3(d=725 \mathrm{pc})$, and a reddening of $E(B-V)=0.08$, as determined by BJG05. We see, unsurprisingly, that the $400 \mathrm{Myr}$ and $500 \mathrm{Myr}$ isochrones are essentially identical redward of the A-type stars. It is also evident from Fig. 3 that the newer isochrones indeed arguably follow the cluster's main sequence somewhat better for redder colors than the earlier one (YY; Yi et al. 2001).

\section{Variability}

The rotational variability and associated periods of the cluster stars provide the principal motivation for our study. This goal requires the sustained acquisition of high-quality imaging data over a sufficiently long time baseline. We are particularly fortunate with respect to the baseline because ours is $\gtrsim 2$ months long. This is four times longer than our conservative expectation of $\sim 15 \mathrm{~d}$ for the longest periods in our color range for a
Hyades-aged cluster, allowing multiple phases to be detected for all rotation periods.

Because our robotic STELLA telescopes are able to acquire data during both good and suboptimal conditions at no additional cost, it is correspondingly necessary for us to discard the latter from among the images acquired by the telescope. We have therefore rejected images with bad seeing, those with very elongated PSFs (usually caused by tracking errors), those affected by lunar proximity, and finally any frames where fewer than $80 \%$ of the stars on our reference images were detected. Frames with mean deviations greater than 0.2 mag from these best exposures were also discarded. Most of the last category consist of images acquired under non-photometric conditions, as revealed by our weather monitors and standard star observations. For this particular observing campaign, the final usable high-quality images constituted $\sim 40 \%$ of the total acquired. Fortunately, this selection does not introduce any significant gaps in the time series beyond a lunar proximity issue centered on 11 Mar. 2014, and minor weather-related interruptions, mostly in April 2014. (The light curve for one of our solar-mass stars, displayed in Fig. 4, demonstrates this continuity in our observations.)

The aperture photometry from the individual exposures was corrected to a common system defined using the best images by cross-identifying $>1000$ well-measured stars with $10<V<16$ over all four fields and across all the exposures. These stars were used as photometric references for making the frame-to-frame corrections relative to the best exposures, allowing the construction of individual light curves, which were then constructed for all cross-identified stars in the field of view of the cluster. We decided to concentrate our efforts on candidate photometric cluster members. Consequently, we then extracted the light curves of 1300 stars along the cluster sequence for careful analysis. This is a superset of the cluster members of BJG05 with $B-V>0.40$, which is the color range of interest in this work.

All light curves were subjected to multiple methods of frequency analysis to identify periodicity - phase dispersion minimization (PDM; Stellingwerf 1978), CLEANed Fourier analysis (Roberts et al. 1987), and generalized Lomb-Scargle periodogram (Zechmeister \& Kurster 2009). While none of these is completely satisfactory in all cases, we have found that for our dataset the CLEAN algorithm appears to be the best one overall. Lomb-Scargle and PDM are affected more often by the $1 \mathrm{~d}$ or multiple period alias. We believe that this behaviour partly comes from our long baseline, during which there is sometimes significant spot evolution, the onset/decline of spot activity, and in certain cases multiple spot groups. CLEAN seems to be less sensitive than the other methods to these problems, piles up power at the rotational frequency, and also allows the window function to be taken into account.

However, there seems to be no substitute for manual inspection of the candidate rotators, taking the raw light curve into account, the results from all three periodicity indicators discussed above and, finally, the phased curves. We found $5 \sigma$ of the noise level in the CLEAN spectra to be a good threshold for accepting periodicity and have generally adopted this as the criterion for a high-quality period (Quality flag $=1$ ). However, it cannot be an automatically adopted criterion, so we made the ultimate decision manually, and on this basis also listed certain lower quality periods (Quality flag =2), where there might be some chance of the listed period being an alias. There are therefore some stars with higher peaks that are assigned Quality flag $=2$, while the rest are considered high quality. On this basis, we have presented 62 rotation periods, of which 8 are listed with Quality flag $=2$. Experiments show that even our $Q=2$ periods have a 

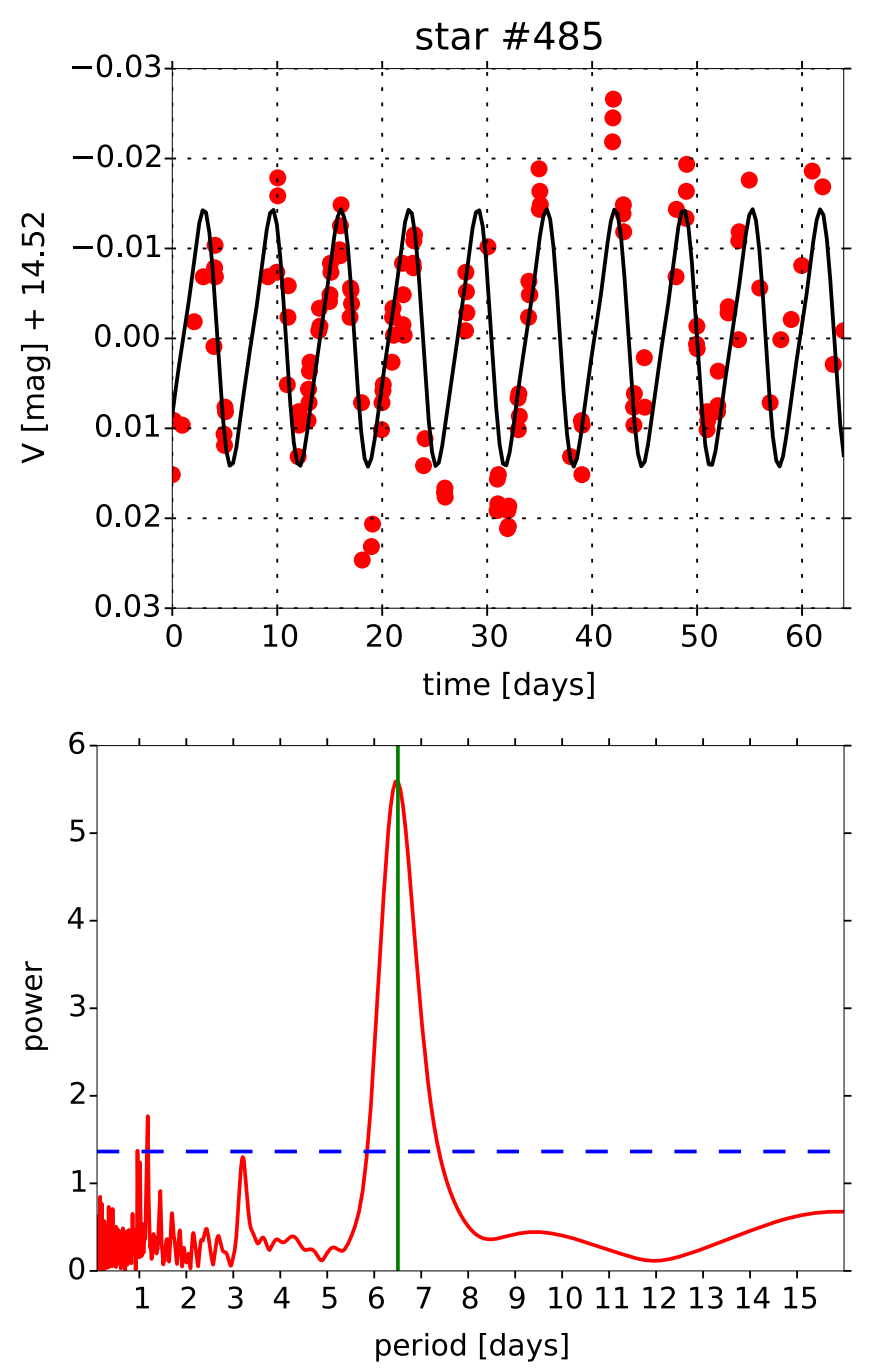

Fig. 4. Upper panel: light curve for star No. 485, a solar-mass star with $(B-V)_{0}=0.664$ in our sample. A periodicity of $6-7 \mathrm{~d}$ is evident from inspection of the light curve. Successive data points show that the photometry for this particular star is clearly repeatable at a level better than 0.01 mag. Our other solar-mass rotators have smaller amplitudes of variability. Lower panel: power spectrum for star No. 485, showing an unambiguous periodicity of the signal at $P=6.53 \mathrm{~d}$, identified as the rotation period of the star.

Scargle false alarm probability, FAP $<0.01$, i.e., a confidence level greater than $99 \%$.

The light curve for a good example of a solar-mass periodic variable is displayed in the upper panel of Fig. 4. The locations of successive data points in the light curve show that the photometry for this particular star is clearly repeatable to a level better than $0.01 \mathrm{mag}$. A periodicity of $6-7 \mathrm{~d}$ is obvious in Fig. 4 . Thanks to the density of our observations, simply plotting the unphased light curve usually allows us to verify the approximate periodicity by visual inspection, as the upper panel of Fig. 4 shows.

The corresponding Fourier power spectrum for this star, constructed using the CLEAN algorithm of Roberts et al. (1987) is displayed in the lower panel of Fig. 4. The peak is at $6.53 \mathrm{~d}$, which is consequently listed as the rotation period of the star. (Light curves, power spectra, and phased light curves for all the periodic candidate cluster rotational variables identified in this study are displayed in the online Appendix to this paper in Figs. B.1-B.9.) We note that few stars actually need to be phased

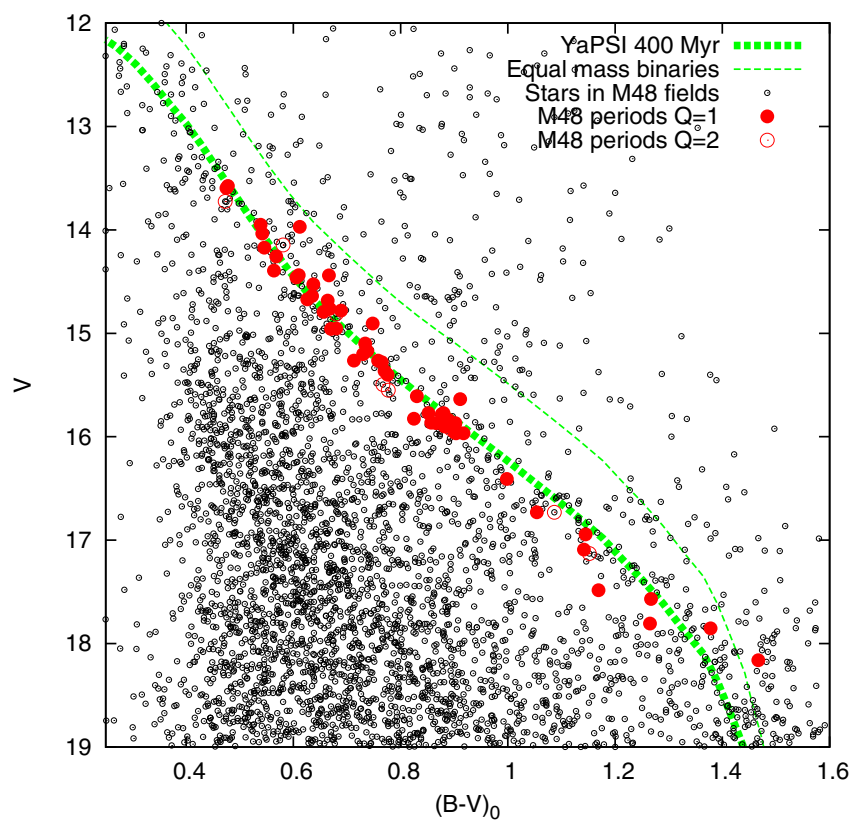

Fig. 5. Color-magnitude diagram for M 48 with the 62 periodic cluster rotational variables highlighted. Solid symbols indicate the 54 highquality $(Q=1)$ periods, and unfilled symbols the 8 lower quality $(Q=2)$ ones. All the periodic cluster member candidates are located on, or relatively near, the cluster sequence. A $400 \mathrm{Myr}$ YaPSI isochrone and the corresponding equal-mass binary sequence are also displayed.

before their periodicity becomes obvious ${ }^{5}$. The periods derived range from $1.67 \mathrm{~d}$ to $13.3 \mathrm{~d}$, the latter well within the sensitivity of our observational window of $\sim 2$ months. These 62 periods are listed in Table A.1, along with other relevant information for each star. This includes the rotation period, its error (the HWHM of the CLEAN peak), the variability amplitude (from sinusoidal fits to the phased light curves), cross-identification with BJG05, the relevant membership indicator, and relevant notes. The periodic stars considered to be cluster members are indicated in the CMD displayed in Fig. 5.

\subsection{Empirical comparisons of the color-period diagram}

The rotation periods derived by the methods described above have then been associated with the photometry discussed earlier in the paper, and the corresponding CPD has been constructed for the cluster stars, as displayed in Fig. 6. This diagram is populated by 62 stars with $(B-V)_{0}$ color range $0.47-1.47 \mathrm{mag}$, and the period range $1.67-13.1 \mathrm{~d}$. The field contamination from the background, as seen in the CMD in Fig. 2 suggests that only a couple of these could possibly be non-members.

The bluer (warmer) half of the M 48 CPD displays a clear sequence of stars ranging from short-period $\sim 2 \mathrm{~d}$ stars at $(B-V)_{0} \sim$ 0.45 to $\sim 8 \mathrm{~d}$ periods at $(B-V)_{0} \sim 0$. 9 . This is reminiscent of the situation in the $625 \mathrm{Myr}$-old Hyades cluster, as discussed below. There is no evidence of the $\mathrm{C} /$ fast sequence of stars with $P \sim 1 \mathrm{~d}$ across the entire $0.5<(B-V)_{0}<1.5$ color range, characteristically seen in younger open clusters such as the Pleiades (Hartman et al. 2010) or M 35 (Meibom et al. 2009).

5 Our time baseline is long enough for significant spot evolution. Correspondingly, our phased light curves would look significantly better "cosmetically" had we suppressed epochs of low stellar variability. They would also look better had we rephased the light curves to the PDM values, within the error envelope of the CLEAN values. 


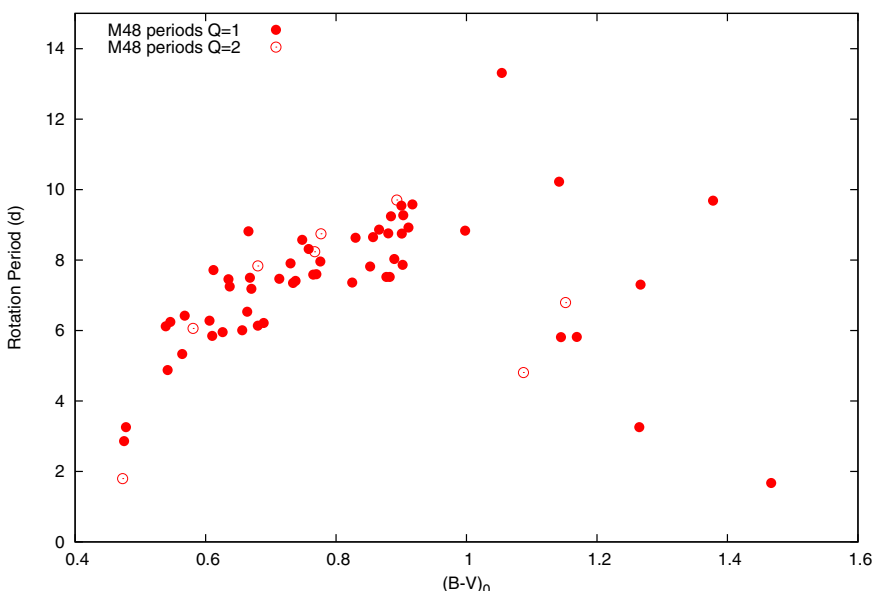

Fig. 6. Measured color-period diagram (CPD) for M 48, showing the 62 periodic stars believed to be cluster rotators, and whose positions in the CMD are displayed in Fig. 5. We observe a relatively distinct sequence of stars, reminiscent of the Hyades sequence, blueward of $B-V=1$, and a more scattered distribution of stars redward of this color. The solar-mass stars show no evidence of the $\mathrm{C} /$ fast sequence that characterizes ZAMS clusters.

This morphology immediately tells us that M48 is older than M 35 and the Pleiades, without even having to perform a detailed comparison.

A more detailed empirical idea of how the M 48 cluster fits in with other cluster observations can be obtained by comparing the constructed M48 CPD directly with other open cluster CPDs. Our first empirical comparison is made with the open cluster M 34, as displayed in the top panel of Fig. 7. The filled and unfilled symbols for M 34 represent the rotation periods determined by Meibom et al. (2011a) and James et al. (2010). Following the original publications, the M34 data have been de-reddened by $E(B-V)=0$. 07 (Canterna et al. 1979). We see that M 48 does not possess the fast G- and K-type rotators blueward of $(B-V)_{0}=1$ that collectively form an (admittedly ill-defined) fast/C sequence in M 34 .

The M 48 slow/I sequence is clearly above that of M 34, indicating that M 48 is older than 250 Myr, the nominal age of M34 (Ianna \& Schlemmer 1993). The I sequences of the two clusters almost overlap at $(B-V)_{0}=0.9$, suggesting that it is already time to move beyond models where the dependence of rotation period, $P$, on age and mass is separable (e.g., Barnes 2003, 2007; Mamajek \& Hillenbrand 2008; Meibom et al. 2011a; Angus et al. 2015).

The corresponding comparison for the Hyades open cluster is displayed in the middle panel of Fig. 7, using the rotation period measurements (filled symbols) of Radick et al. (1987), and the more recent measurements (unfilled symbols) of Delorme et al. (2011). Taylor (2006) has determined that the Hyades reddening is $E(B-V) \leq 0$. $01 \mathrm{mag}$, and consequently the $B-V$ values from neither of these studies have been dereddened.

We see that the rotation periods of similar-mass stars in M 48 and in the Hyades are comparable, indicating that the ages of the two clusters are roughly comparable. (In their comprehensive study of the Hyades, Perryman et al. 1998, derived an age of 625 Myr.) Closer inspection shows that the average M 48 rotational sequence in the CPD is clearly below the average of the Hyades sequence, telling us that M 48 is somewhat younger. The average difference between the two sequences in the wellsampled $0.5<(B-V)_{0}<0.9$ interval is $0.99 \mathrm{~d}$.
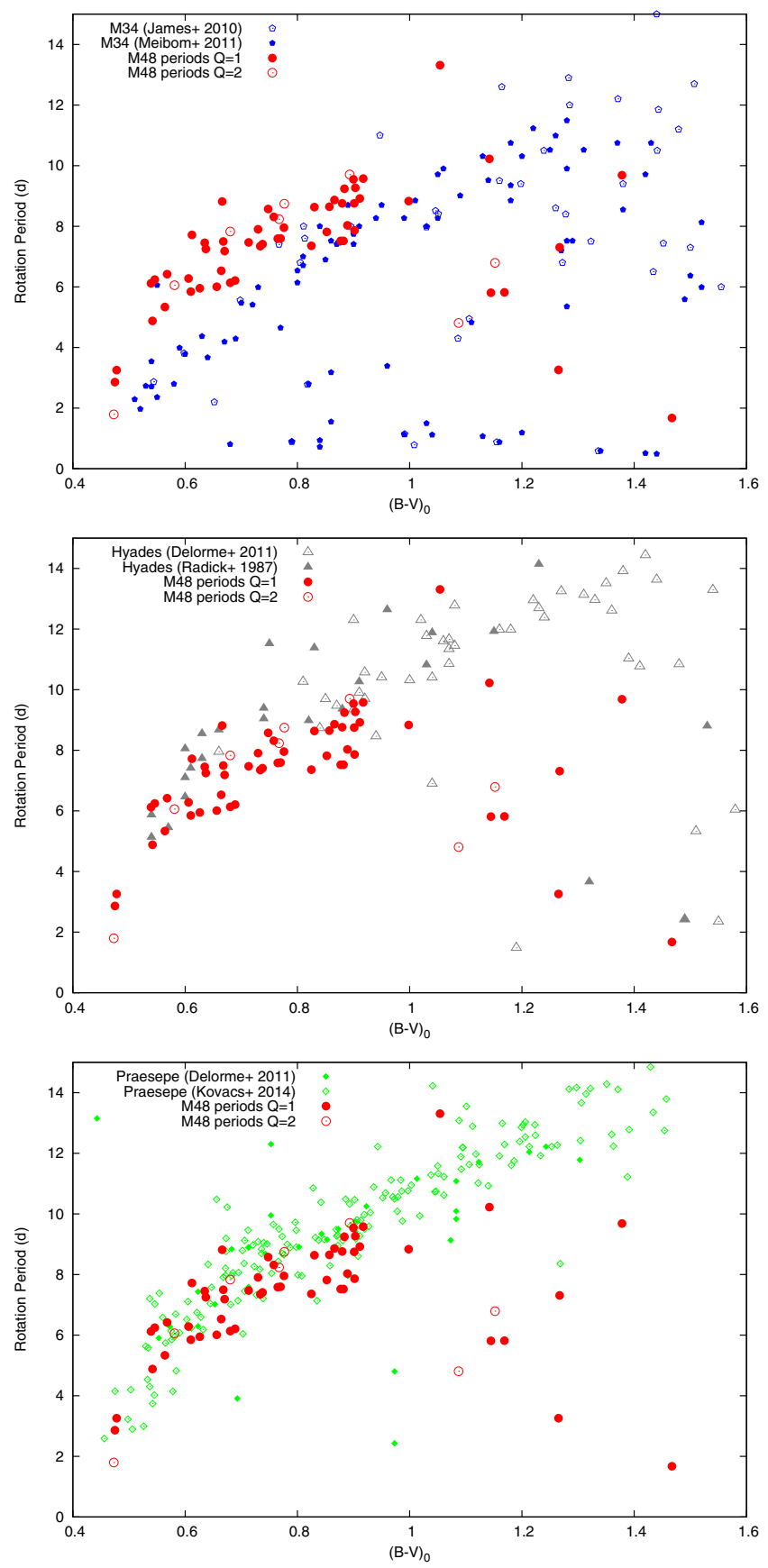

Fig.7. Top panel: comparison of the M 48 CPD (circles, red online) with that of M 34 (250 Myr old; pentagons, dark blue online). We see that the M48 sequence is clearly above that of M34, indicating that M 48 is older. Another indicator of M 48's older age is it's lack of fastrotating stars, which form a sparsely populated sequence in M 34 (with $P \sim 1 \mathrm{~d}$ and $\left.0.6<(B-V)_{0}<1\right)$. Middle panel: comparison of the M 48 $\mathrm{CPD}$ with that of the Hyades cluster (triangles, gray online). While the rotation periods of similar-mass stars are comparable between the two clusters, indicating that their ages are roughly comparable, the average solar-type M 48 star (see text) is $0.99 \mathrm{~d}$ below that for the $625 \mathrm{Myr}$-old Hyades, indicating that M 48 is somewhat younger. Bottom panel: comparison of the M 48 CPD with that of the 590 Myr-old Praesepe cluster (diamonds, green online), confirming that M 48 is again younger. The average solar-type M 48 star (see text) is closer $(0.44 \mathrm{~d})$, consonant with Praesepe's age being slightly lower than that of the Hyades.

An empirical confirmation of this result is available using the rotation period determinations for the Praesepe cluster by Delorme et al. (2011) and Kovacs et al. (2014). These are 


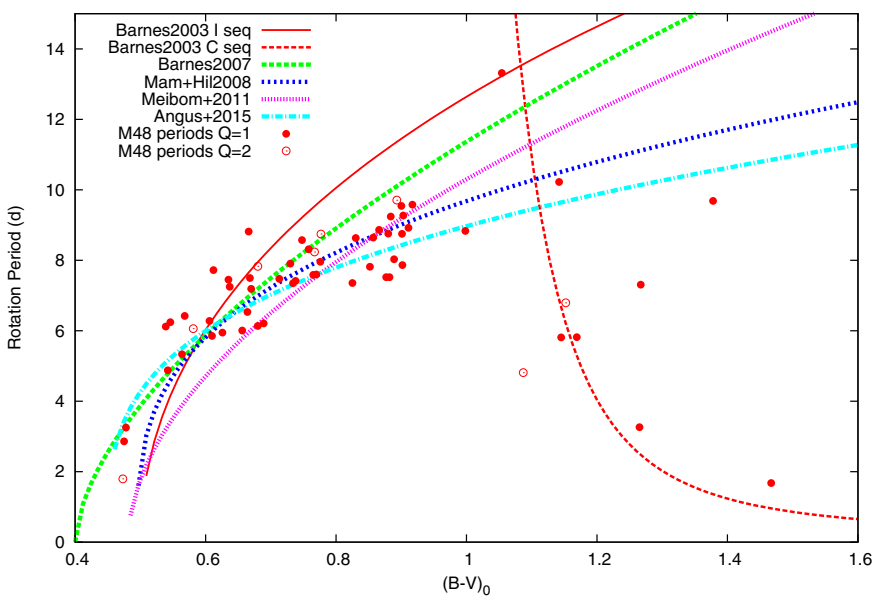

Fig. 8. Comparison of the M $48 \mathrm{CPD}$ with rotational isochrones for models with separable $(t, M)$ dependencies, constructed for an age of 400 Myr. The M 48 data points have been de-reddened by 0.08 . These models do not satisfactorily capture the detailed morphology of the observations (see text for details).

plotted in the bottom panel of Fig. 7 using filled and unfilled symbols respectively. In accordance with the determination of Taylor (2006), the $B-V$ color values for Praesepe have been de-reddened by $E(B-V)=0$. 027 . Praesepe is known to be of very similar age (590 Myr; Fossati et al. 2008) to the Hyades. Our comparison of the M48 and Praesepe CPDs is consonant with this result. In fact, after comparing the Praesepe and Hyades CPDs, Delorme et al. (2011) concluded that Praesepe is slightly younger than the Hyades, perhaps by about $50 \mathrm{Myr}$. Our comparison in Fig. 7 seems to confirm this conclusion in that the Praesepe sequence is indeed slightly closer to the M 48 sequence than that of the Hyades. The average difference between the two sequences in the $0.5<(B-V)_{0}<0.9$ interval is $0.44 \mathrm{~d}$, smaller than the Hyades-M48 difference of $0.99 \mathrm{~d}$. These empirical comparisons tell us that the rotational age of M 48 is almost certainly in the $(250,590)$ Myr interval.

\subsection{Comparison against models with separable (t, M) dependencies}

These new M 48 rotation periods also permit a useful comparison with prior predictions for the locations of stars of its age in the CPD. Figure 8 shows how these data compare with a number of empirical studies that relied on the rotation period $P$ having separable dependencies on age, $t$, and stellar mass $M$ (or a suitable mass proxy such as color) - the original gyrochronology formulation of Barnes (2003), the update in Barnes (2007), the modification to that formulation proposed by Mamajek \& Hillenbrand (2008), one based largely on the M 34 cluster data of Meibom et al. (2011a), and the recent one of Angus et al. (2015), the last based on a very limited cluster dataset, and a small number of field stars with ages determined from asteroseismology. The displayed comparisons all use the prior published age for M 48 of 400 Myr. Moderate changes to the age used make moderate changes to the displayed curves, but do not change the overall behavior.

We observe that all the models displayed here capture, to a certain extent, the overall behavior of the M 48 data blueward of $(B-V)_{0} \sim 1$. However, there is a potentially serious problem redward of this color value. The measured rotation periods seem to decline for redder (lower-mass) stars. To a certain extent, this behavior is not unexpected, because these empirical formulations of gyrochronology explicitly ignored the fast rotators (with the exception of Barnes (2003), which treated them separately as shown in Fig. 8), electing to concentrate attention on the slow/I sequence stars, which are the only ones seen in M 48 blueward of $(B-V)_{0}=1$.

There are also certain differences, obvious in Fig. 8, with respect to the point where these models intersect the color axis, and with the color range over which each of these provides a good fit (or not) to the periods. (We will show a detailed comparison with a subsequent preferred model below.) Finally, it should be noted that the separable empirical models have slightly differing dependencies on the age, a fact that is imperceptible in such a comparison, being overwhelmed by the mass dependence.

We have not been able to identify any red stars (say $(B-$ $\left.V)_{0} \gtrsim 1\right)$ that are very slow rotators $(P>10 \mathrm{~d})$. While our observations do possess the time baseline to identify such stars, any such stars are fainter than $V=16.5$ (see Fig. 5), where the precision of our photometry declines, affecting our sensitivity to such periods if their variability amplitudes are small. Our data therefore do not allow us to state conclusively whether such stars actually exist or not in M 48 .

\subsection{Comparison with the B2010 model}

Our preferred way of interpreting these M48 rotation period data is with the gyrochronology formulation of Barnes (2010), hereafter B10, based on the rotational evolution ideas described in Barnes \& Kim (2010). A significant part of this preference arises from the simplicity of this model, where only two dimensionless constants, $k_{\mathrm{C}}$ and $k_{\mathrm{I}}$, are required to specify the two relevant spindown timescales for all fast and slow cool stars, and hence of the entire main sequence rotational evolution of cool stars. The B10 formulation describes the behaviors of both the slow/I-type stars and the fast/C-type stars in a single model, treating them symmetrically ${ }^{6}$. Furthermore, the rotational isochrones constructed using this model are better than those from other models in matching the measured colorperiod diagram of the 2.5 Gyr-old cluster NGC 6819 , studied by Meibom et al. (2015). And we show below that this model describes the morphology of the M 48 CPD in considerable detail.

This B10 model provides the age of an individual star as an explicit function of its rotation period (and a mass variable), albeit with more complexity than the separable empirical models discussed above. One can then take a suitable average over the cluster stars to derive the corresponding cluster age. The procedure first requires the calculation of the (global) convective turnover timescale, $\tau$, for each star from its de-reddened $B-V$ color. This is simply accomplished by interpolation from Table 1 in Barnes \& Kim (2010). Then one applies Eq. (32) from B10,

$t=\frac{\tau}{k_{\mathrm{C}}} \ln \frac{P}{P_{0}}+\frac{k_{I}}{2 \tau}\left(P^{2}-P_{0}^{2}\right)$.

This is an explicit expression for the age $t$ of the star, in terms of its measured rotation period $P$, and $\tau$, the latter a proxy for its mass or color. Here, $k_{\mathrm{C}}=0.646 \mathrm{Myr} / \mathrm{d}$ and $k_{\mathrm{I}}=452 \mathrm{~d} / \mathrm{Myr}$ are two dimensionless constants whose values are decided by the totality of the open cluster rotation period data, and the solar datum; we have simply adopted unchanged the values proposed in B10, particularly in view of the model's ability to reproduce well the CPD of the 2.5 Gyr-old cluster NGC 6819, as discussed in Meibom et al. (2015). $P_{0}$ is the initial rotation period, set to

6 Brown (2014) has called this the "Symmetric Empirical Model". 


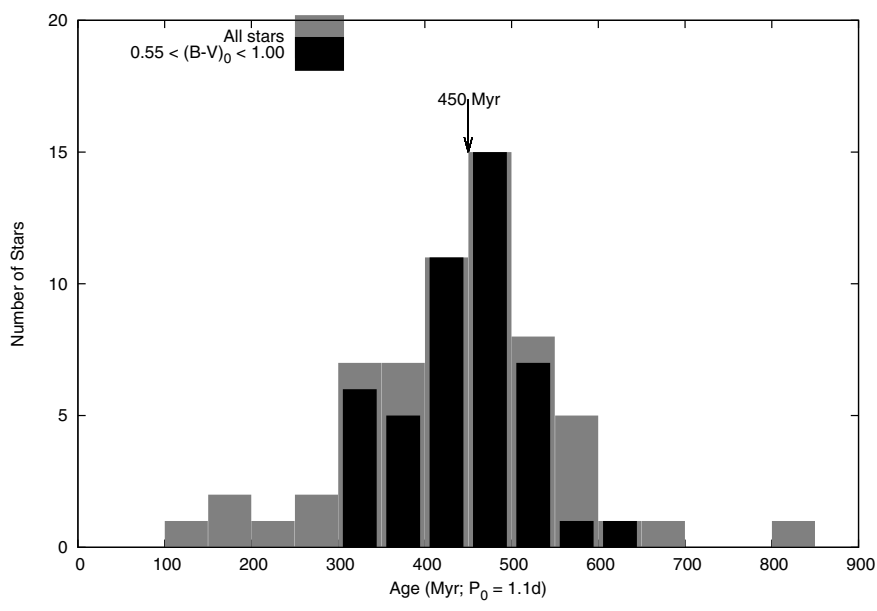

Fig. 9. Histograms of gyro-age for the individual M48 stars, calculated using the B10 rotational model and assuming an initial period of $1.1 \mathrm{~d}$. The gray histogram shows all 62 stars, while the black histogram is for a sample restricted to the 46 stars in the particularly wellmeasured $0.55<(B-V)_{0}<1.00$ region. The larger sample gives a median age of $451 \mathrm{Myr}($ mean $=441 \mathrm{Myr}$, S.D. $=117 \mathrm{Myr}=26 \%$ ), and the smaller one gives similar values, with a median age of $457 \mathrm{Myr}$ $($ mean $=447 \mathrm{Myr}$, S.D. $=70 \mathrm{Myr}=16 \%$.) This large age dispersion mostly arises from having to assume a single initial period, $P_{0}(=1.1 \mathrm{~d})$ for all stars.

$P_{0}=1.1 \mathrm{~d}$, following B10, but we will also allow it to vary within certain bounds below, as allowed by observations, and as described in B10. (It is also possible to use other turnover timescales, but then one must be careful to recalibrate the constants $k_{\mathrm{C}}$ and $k_{\mathrm{I}}$ to match the totality of the open cluster and solar rotational data, as discussed in $\mathrm{B} 10$.)

Provided that the measured period is greater than $P_{0}$, each measured rotation period $P_{i}$ results in a corresponding gyroage $t_{i}$, which is plotted in the histograms in Fig. 9 for $P_{0}=$ $1.1 \mathrm{~d}$. The gray histogram shows the distribution for all 62 stars with measured rotation periods. We obtain a distribution that is sharply peaked at a median value of $451 \mathrm{Myr}$, and mean value of 441 Myr but with relatively wide wings, giving a standard deviation of $117 \mathrm{Myr}(=26 \%)$.

A significant portion of this relatively large dispersion can be traced to a number of outliers. For instance, the outlier at $t_{i}=840 \mathrm{Myr}$ is No.1456, the one above all the other stars in the CPD, with $\left(P,(B-V)_{0}\right)=(13.3 \mathrm{~d}, 1.054)$. Because it lies on the cluster sequence in the CMD, there is no good reason to discard it at the present time. The other outliers are mostly either very blue or very red stars (defined for this study as lying outside the $0.55<(B-V)_{0}<1.00$ color range).

The black histogram in Fig. 9 shows the distribution for the 46 stars in this restricted $0.55<(B-V)_{0}<1.00$ color range, where the I sequence is particularly well-defined. This color region contains the Sun and many well-studied open clusters, and is known from the rotation period observations for NGC 6819 (2.5 Gyr) by Meibom et al. (2015) to be well-calibrated. In the corresponding histogram, many of the outliers disappear to reveal a distribution where $38 / 46=83 \%$ of the stars are confined to the $t=[350,550]$ Myr region. An M 48 cluster gyro-age outside this range is essentially ruled out. The formal mean age for this restricted distribution is $\bar{t}=447 \mathrm{Myr}$ (median $=457 \mathrm{Myr}$ ), but with a smaller standard deviation $\sigma_{t}=70 \mathrm{Myr}(=16 \%)$. These results enable us to confirm the $400 \pm 100 \mathrm{Myr}$ isochrone age of this cluster, and to propose a mean rotational (gyrochronology) age of 450 Myr for M 48.

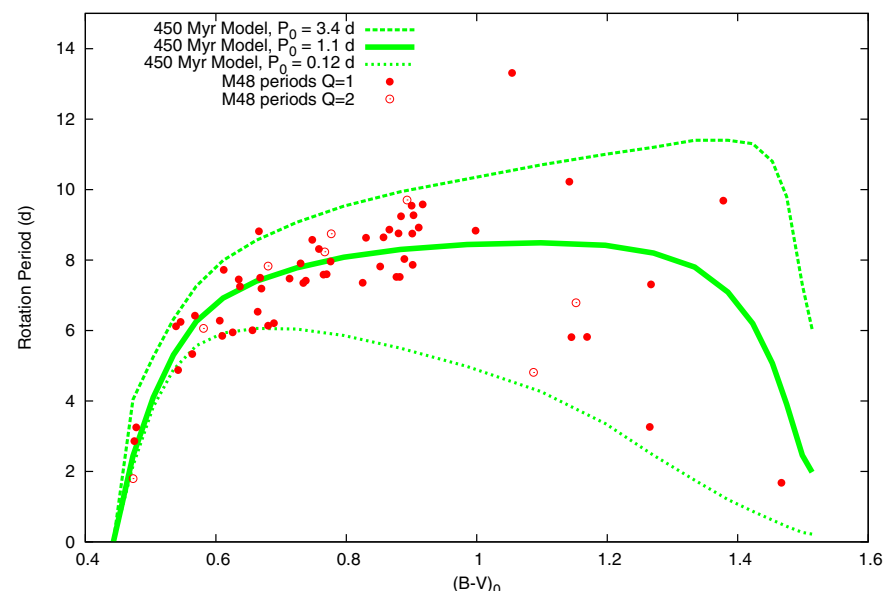

Fig. 10. Theoretical rotational isochrones for $450 \mathrm{Myr}$, constructed following Barnes (2010), are compared with the M48 CPD. The three curves from top to bottom respectively correspond to initial periods of $3.4 \mathrm{~d}, 1.1 \mathrm{~d}$, and $0.12 \mathrm{~d}$, representing the range allowed by ZAMS cluster observations. Almost all the data points are consistent with a single rotational age of $450 \mathrm{Myr}$.

We now show that much of the above scatter arises from intrinsic astrophysical variations in the initial rotation periods, so that it is likely that the uncertainty on the gyro-age of M48 is actually significantly smaller than the 70 Myr standard deviation for the best-measured stars. For open clusters that are significantly younger than the Hyades, it is well-known, and expected, that the rotation periods of cool stars will not necessarily have converged to a single sequence. Indeed, in ZAMS clusters, two distinct sequences are sometimes observed, as discussed earlier, and as is visible in the CPD for M 34 (see Fig. 7). In the M 48 $\mathrm{CPD}$, stars with masses greater than $0.9 M_{\odot}\left(B-V_{0}<1.0\right)$ have essentially converged to a single sequence, while lower mass stars most certainly have not.

There is a simple way to understand the dispersion in the age distribution derived above. Given an age $t$, and using a given initial period $P_{0}$, one can solve equation (1) numerically ${ }^{7}$ to associate a value $P$ to every $\tau$ value of interest, or equivalently the value of $(B-V)_{0}$. Such a curve is the isochrone for that initial period, since it is the locus of all such equal-age points. The $450 \mathrm{Myr}$ isochrone for $P_{0}=1.1 \mathrm{~d}$ is displayed in Fig. 10 with the thick central line (solid green). Barnes (2010) found that the lower- and upper envelopes of the initial period distribution could be reasonably set at $0.12 \mathrm{~d}$ and $3.4 \mathrm{~d}$ respectively. Carrying out the corresponding calculations for $P_{0}=0.12 \mathrm{~d}$ and $P_{0}=3.4 \mathrm{~d}$ with the same age of $450 \mathrm{Myr}$ yields the lower (dotted green) and upper lines (dashed green) respectively. (Other intermediate initial period values in this range would provide curves that are bounded by these.)

This collection of curves can be viewed as representing the total rotational isochrone for M 48, providing a narrow sequence at the blue/solar-like end, and a wider sequence for lower mass stars. We observe that this range of initial conditions explains almost all the scatter in the rotation period measurements as a function of mass, because only one of our 62 rotation periods lies significantly outside the range of these isochrones. Consequently, with $P_{0}$ allowed to vary within the astrophysical limits permitted by ZAMS observations, almost all our stars are

7 For a given $P, P_{0}$, and age $t$, one can also obtain a solution for $\tau$ analytically, as shown in Barnes (2010), by simply solving a quadratic equation. 


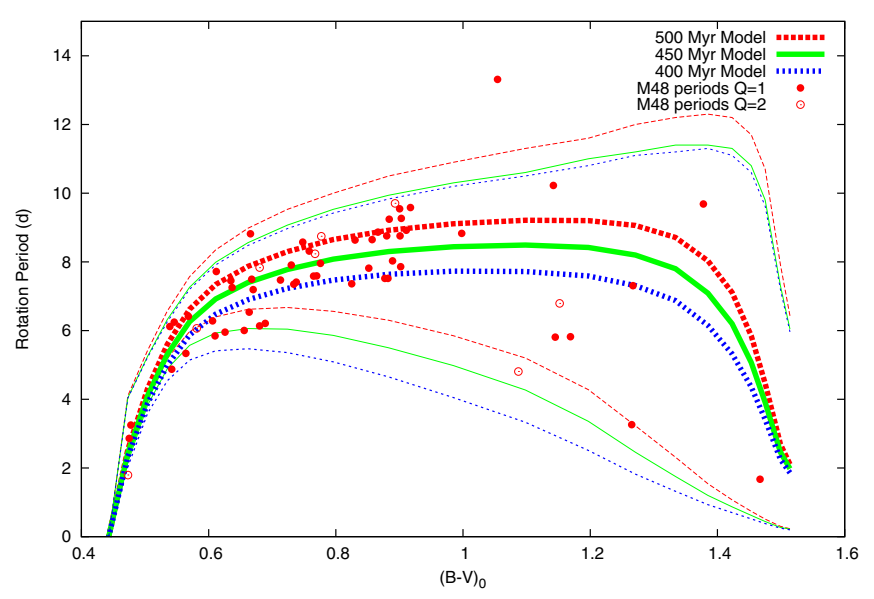

Fig. 11. CPD for M 48 compared with younger (400 Myr; dotted, blue online) and older (500 Myr; dashed, red online) rotational isochrones. Neither the $400 \mathrm{Myr}$ nor the $500 \mathrm{Myr}$ isochrone provide a good match to the data, passing significantly below or above the core group of stars with $0.6<(B-V)_{0}<0.9$. The cluster's rotational age is clearly within this $(400,500) \mathrm{Myr}$ interval. (For clarity, the (central) $P_{0}=1.1 \mathrm{~d}$ isochrones for the three separate ages are emphasized.)

consistent with a single age of $450 \mathrm{Myr}$. This fact is consistent with the current belief that open clusters are simple stellar populations, and that they are describable as single-age populations.

We now ask how well we know the gyro-age of the cluster as a whole. The histogram of ages (displayed in Fig. 9) shows that moving $\pm 50 \mathrm{Myr}$ off the mean age halves the bin occupancies. Bins beyond these ages are occupied by stars for which $1.1 \mathrm{~d}$ is not a good estimate for the initial period, as Fig. 10 shows. We therefore construct $400 \mathrm{Myr}$ and $500 \mathrm{Myr}$ rotational isochrones, and display them in Fig. 11 (dotted blue and dashed red, respectively), in an analogy with classical isochrones. Neither of these can be considered a reasonable fit to the M48 CPD. In particular, the $400 \mathrm{Myr}$ and $500 \mathrm{Myr}$ isochrones for $P_{0}=1.1 \mathrm{~d}$ pass below and above the core of the rotation period distribution with $0.6<(B-V)_{0}<0.9$. The $P_{0}=3.4 \mathrm{~d}$ isochrones are not particularly informative. However, the $P_{0}=0.12 \mathrm{~d}$ isochrones for 400- and 500 Myr also pass respectively below and above the group of the fastest cluster rotators at near-Solar color (and maybe even redder values), making them significantly worse fits to the fast edge of the rotation period distribution. We therefore consider $50 \mathrm{Myr}$ to be a reasonable estimate of the uncertainty on the gyro-age of M 48, which we suggest is $450 \pm 50 \mathrm{Myr}$.

Another approach is the following. Ignoring their individual membership of M 48 and treating all the 62 measured stars independently gives the histogram displayed in Fig. 9, with its standard deviation of 117 Myr. Clearly, the uncertainty on the mean age of the cluster must be much lower than the uncertainty of an individual star. The standard error (S.E.) of the mean is formally $\sigma_{t} / \sqrt{\text { Number of stars }}=117 / \sqrt{62} \approx 15$ Myr. But taking this to represent the error in cluster age seems unjustified, in view of the possibility of residual systematic age errors arising from our incomplete understanding of rotational stellar evolution.

To estimate possible systematic errors, we constructed equivalent age distributions for the comparison clusters displayed in Fig. 7, again using the $0.55<(B-V)_{0}<1.0$ interval. These give mean ages of 260, 522, and 584 Myr for M 34, Praesepe, and the Hyades, respectively, which suggest that if the corresponding isochrone ages of 250, 590, and $625 \mathrm{Myr}$, are taken as absolute truth, then systematic errors in the B10 version of gyrochronology could contribute at the level of $\sim 40 \mathrm{Myr}$ to the M 48 age uncertainty. Then adding, in quadrature, the $15 \mathrm{Myr}$ internal error of the mean for M 48 gives 44 Myr. This suggests that the \pm 50 Myr value quoted above is not unreasonable.

In closing, it is worth noting that an analysis like the one above might not be as easily accomplished for a much younger cluster, where the lower-mass stars are still on the pre-main sequence (and the demands on rotational evolution models are more severe). Indeed Cargile et al. (2014) appear to have experienced some difficulties in adapting the Barnes (2010) formulation for the $\sim 150 \mathrm{Myr}$-old Blanco 1 open cluster, and resorted to the separable $(t, m)$ formulations for $P=P(t, m)$ to interpret that cluster's CPD.

\section{Conclusions}

We have performed a two-month-long photometric time series campaign on the southern open cluster M 48 (NGC 2548) using the AIP's STELLA I robotic imaging telescope and associated WiFSIP 4K imager, located in Tenerife. We also acquired photometry in the Johnson $B$ and $V$ bands to a depth of $V \sim 20$ for the $44^{\prime} \times 44^{\prime}$ region centered on the cluster. A relatively clear cluster sequence is visible in our photometry and largely coincides with the astrometric and photometric candidate members identified by prior work on the cluster. This sequence in the CMD is followed closely by a theoretical isochrone and is also closely matched by the rotational variables.

We constructed light curves populated with $\sim 120$ data points each and with no serious data gaps over the two-month observing baseline. Our time-series photometry is repeatable at the 3 mmag level for F-G stars, with the uncertainty increasing as expected for fainter stars. We successfully derived rotation periods for $62 \mathrm{cool}$ photometric cluster member stars, 54 of which are classified as higher-quality, and 8 are lower-quality. These rotation periods and the associated colors of the stars define the M 48 cluster's CPD, the rotational equivalent of the CMD. In the CPD, these periods delineate a clear sequence blueward of $(B-V)_{0}=1.0$; redward of this point, the rotation period distribution has a significantly larger scatter that is likely astrophysical.

While comparable with the rotational sequences in the $625 \mathrm{Myr}$-old Hyades open cluster and the 590 Myr-old Praesepe cluster, this sequence lies below both, empirically demonstrating that M 48 is younger. Likewise, direct comparison with the CPD for the 250 Myr-old M 34 open cluster shows that M 48 is older.

We constructed the distribution of gyro-ages for the cluster stars, finding one that is sharply peaked at $450 \mathrm{Myr}$, but with relatively wide wings, giving a standard deviation of $117 \mathrm{Myr}$ $(=26 \%)$, and suggesting that this precision for comparable field star rotators is reasonable and attainable.

We showed that the known (astrophysical) contribution from initial period variations on the ZAMS accounts for much of the scatter in the rotational ages, so that almost all the measured rotation periods are actually consistent with a single cluster age of 450 Myr. For the cluster as a whole, the age uncertainty is about 50 Myr. We therefore propose a mean cluster age from gyrochronology for M 48 of $450 \pm 50$ Myr.

In sum, this study has added another open cluster of intermediate age to the canonical literature.

Acknowledgements. This work was supported by the German Deutsche Forschungsgemeinschaft, DFG project number DFG STR645/7-1. This work is based on data obtained with the STELLA robotic telescopes in Tenerife, an AIP facility jointly operated by AIP and IAC. Detailed comments from an anonymous referee are also appreciated. 


\section{References}

Allard, F., Homeier, D., \& Freytag, B. 2011 in 16th Cambridge Workshop on Cool Stars, Stellar Systems, and the Sun, eds. C. Johns-Krull, M. Browning, \& A. West, ASP, 448, 91

Angus, R., Aigrain, S., Foreman-Mackey, D., \& McQuillan, A. 2015, MNRAS, 450,1787

Balaguer-Nunez, L., Jordi, C., \& Galadi-Enriquez, D. 2005, A\&A, 437, 457 (BJG05)

Baliunas, S. L., Sokoloff, D., \& Soon, W. H. 1996, ApJ, 457, L99

Barnes, S. A. 2003, ApJ, 586, 464

Barnes, S. A. 2007, ApJ, 669, 1167

Barnes, S. A. 2010, ApJ, 722, 222 (B10)

Barnes, S. A., \& Kim, Y.-C. 2010, ApJ, 721, 675

Barnes, S. A., \& Sofia, S. 1996, ApJ, 462, 746

Barnes, S. A., Sofia, S., Stauffer, J., \& Prosser, C. P. 1999, ApJ, 516, 263

Bertin, E., \& Arnouts, S. 1996, A\&A, 117, 393

Bouvier, J., Cabrit, S., Fernandez, M., Martin, E. L., \& Matthews, J. M. 1993, A\&A, 272, 176

Bouvier, J., Forestini, M., \& Allain, S. 1997, A\&A, 326, 1023

Brown, T. M. 2014, ApJ, 789, 101

Calabretta, M. R., \& Greisen, E. W. 2002, A\&A, 395, 1077

Canterna, R., Perry, C. L., \& Crawford, D. L. 1979, PASP, 91, 263

Cargile, P., James, D. J., Pepper, J., et al. 2014, ApJ, 782, 29

Chaboyer, B., Demarque, P., \& Pinsonneault, M. H. 1995, ApJ, 441, 876

Chromey, F. R., \& Hasselbacher, D. A. 1996, PASP, 108, 944

Collier Cameron, A., Campbell, C. G., \& Quaintrell, H. 1995, A\&A, 298, 133

Crawford, D. L. 1975, AJ, 80, 955

Delorme, P., Cameron, A. C., Hebb, L., et al. 2011, MNRAS, 413, 2218

Ebbighausen, E. G. 1939, ApJ, 90, 689

Epstein, C., \& Pinsonneault, M. 2014, ApJ, 780, 159

Fossati, L., Bagnulo, S., Landstreet, J., et al. 2008, A\&A, 483, 891

Gallet, F., \& Bouvier, J. 2015, A\&A, 577, A98

Granzer, T. 2004, Astron. Nachr., 325, 513

Hartman, J. D., Gaudi, B. S., Pinsonneault, M. H., et al. 2009, ApJ, 691, 342

Hartman, J. D., Bakos, G. A., Kovacs, G., \& Noyes, R. W. 2010, MNRAS, 408, 475

Hauschildt, P. H., Allard, F., \& Baron, E. 1999, ApJ, 512, 377

Ianna, P. A., \& Schlemmer, D. M. 1993, AJ, 105, 209

Irwin, J., Hodgkin, S., Aigrain, S., et al. 2007, MNRAS, 377, 741

James, D. J., Barnes, S. A., Meibom, S., et al. 2010, A\&A, 515, A100

Kovacs, G, Hartman, J. D., Bakos, G. A., et al. 2014, MNRAS, 442, 2081
Kraft, R. 1970, in Spectroscopic Astrophysics, An Assessment of the Contributions of Otto Struve, ed. G. H. Herbig (Berkeley: University of California Press), 385

Landolt, A. 2009, AJ, 137, 4186

MacGregor, K. B., \& Brenner, M. 1991, ApJ, 376, 204

Mamajek, E., \& Hillenbrand, L. A. 2008, ApJ, 687, 1264

Matt, S., Brun, A. S., Baraffe, I., et al. 2015, ApJ, 799, L23

Meibom, S., Mathieu, R. D., \& Stassun, K. G. 2009, ApJ, 653, 621

Meibom, S., Mathieu, R. D., Stassun, K. G., Liebesny, P., \& Saar, S. H. 2011a, ApJ, 733, 115

Meibom, S., Barnes, S. A., Latham, D. W., et al. 2011b, ApJ, 733, L9

Meibom, S., Barnes, S. A., Platais, I., et al. 2015, Nature, 517, 589

Mink, D. J. 2002, in Astronomical Data Analysis Software and Systems XI, eds. D. A. Bohlender, D. Durand, \& T. H. Handley (San Francisco: ASP), ASP Conf. Proc., 281, 169

Patten, B. M., \& Simon, T. 1996, ApJS, 106, 489

Perryman, M. A. C., Brown, A. G. A., Lebreton, Y., et al. 1998, A\&A, 331, 81

Pesch, P. 1961, ApJ, 134, 602

Queloz, D., Allain, S., Mermilliod, J.-C., Bouvier, J., \& Mayor, M. 1998, A\&A, 335,183

Radick, R. R., Thompson, D. T., Lockwood, G. W., Duncan, D. K., \& Baggett, W. E. 1987, ApJ, 321, 459

Rider, C. J., Tucker, D. L., Smith, J. A., et al. 2004, AJ, 127, 2210

Roberts, D. H., Lehar, J., \& Dreher, J. W. 1987, AJ, 93, 968

Sharma, S., Pandey, A. K., Ogura, K., et al. 2006, AJ, 132, 1669

Sills, A., Pinsonneault, M. H., \& Terndrup, D. M. 2000, ApJ, 534, 335

Soderblom, D. R., Stauffer, J. R., Hudon, J. D., \& Jones, B. F. 1993, ApJS, 85, 315

Spada, F., Demarque, P., Kim, Y.-C., \& Sills, A. 2013, ApJ, 776, 87

Stauffer, J. R., \& Hartmann, L. W. 1987, ApJ, 318, 337

Stellingwerf, R. F. 1978, ApJ, 224, 953

Strassmeier, K. G., Granzer, T., Weber, M., et al. 2004, Astron. Nachr., 325, 527

Strassmeier, K. G., Granzer, T., \& Weber, M. 2010, Adv. Astron., 19

Strassmeier, K. G., Weingrill, J., Granzer, T., et al. 2015, A\&A, 580, A66

Taylor, B. J. 2006, AJ, 132, 2453

van Leeuwen, F., \& Alphenaar, P. 1982, ESO Messenger, 28, 15

van Leeuwen, F., Alphenaar, P., \& Meys, J. J. M. 1987, A\&AS, 67, 483

Wilson, O. C. 1978, ApJ, 226, 379

Wu, Z. Y., Tian, K. P., Balaguer-Nunez, L., et al. 2002, A\&A, 381, 464

Wu, Z.-Y., Zhou, X., Ma, J., Jiang, Z.-J., \& Chen, J.-S. 2006, PASP, 118, 1104

Yi, S., Demarque, P., Kim, Y.-C., et al. 2001, ApJS, 136, 417

Zechmeister, M., \& Kurster, M. 2009, A\&A, 496, 577 
Appendix A: Additional table

Table A.1. Rotation periods of the M 48 stars.

\begin{tabular}{|c|c|c|c|c|c|c|c|c|c|}
\hline Id & $\begin{array}{c}V \\
\mathrm{mag}\end{array}$ & $\begin{array}{c}B-V \\
\text { mag }\end{array}$ & $\begin{array}{c}P \\
\text { days }\end{array}$ & $\begin{array}{l}\text { Perr } \\
\text { days }\end{array}$ & $\begin{array}{l}\text { amp } \\
\text { mag }\end{array}$ & mem & $Q$ & BJG \# & Notes \\
\hline 284 & 13.577 & 0.56 & 3.25 & 0.08 & 0.013 & - & 1 & & Variable in 2nd half \\
\hline 287 & 13.597 & 0.56 & 2.86 & 0.10 & 0.018 & $\mathrm{M}$ & 1 & BJG 3157 & \\
\hline 303 & 13.726 & 0.55 & 1.80 & 0.02 & 0.007 & M & 2 & BJG 841 & Low Amplitude \\
\hline 332 & 13.951 & 0.62 & 6.12 & 0.41 & 0.037 & - & 1 & & \\
\hline 336 & 13.970 & 0.69 & 7.72 & 0.56 & 0.030 & - & 1 & & \\
\hline 343 & 14.034 & 0.62 & 4.88 & 0.22 & 0.015 & M & 1 & BJG 3584 & \\
\hline 363 & 14.146 & 0.66 & 6.06 & 0.42 & 0.017 & M & 2 & BJG 2049 & 2 spot groups, PDM period \\
\hline 368 & 14.172 & 0.63 & 6.24 & 0.35 & 0.022 & - & 1 & & \\
\hline 386 & 14.255 & 0.65 & 6.42 & 0.35 & 0.014 & M & 1 & BJG 2109 & \\
\hline 407 & 14.395 & 0.64 & 5.33 & 0.26 & 0.020 & M & 1 & BJG 3680 & \\
\hline 418 & 14.439 & 0.69 & 5.85 & 0.36 & 0.019 & - & 1 & & \\
\hline 421 & 14.440 & 0.75 & 8.82 & 0.64 & 0.012 & M & 1 & BJG 2037 & \\
\hline 425 & 14.459 & 0.69 & 6.28 & 0.44 & 0.009 & M & 1 & BJG 1614 & \\
\hline 437 & 14.525 & 0.72 & 7.25 & 0.72 & 0.019 & M & 1 & BJG 3583 & \\
\hline 467 & 14.640 & 0.71 & 7.45 & 0.57 & 0.014 & - & 1 & & \\
\hline 482 & 14.671 & 0.71 & 5.95 & 0.32 & 0.029 & M & 1 & BJG 2975 & \\
\hline 485 & 14.683 & 0.74 & 6.53 & 0.42 & 0.028 & M & 1 & BJG 4781 & \\
\hline 501 & 14.762 & 0.75 & 7.50 & 0.52 & 0.018 & M & 1 & BJG 521 & \\
\hline 507 & 14.781 & 0.77 & 6.21 & 0.36 & 0.018 & - & 1 & & \\
\hline 511 & 14.792 & 0.74 & 6.00 & 0.23 & 0.020 & $\mathrm{M}$ & 1 & BJG 3846 & \\
\hline 517 & 14.812 & 0.76 & 7.83 & 1.73 & 0.011 & $\mathrm{M}$ & 2 & BJG 3927 & $P \sim 7 \mathrm{~d}$ possible, $\mathrm{DR} ?$ \\
\hline 540 & 14.904 & 0.83 & 8.57 & 0.72 & 0.012 & $\mathrm{M}$ & 1 & BJG 2600 & \\
\hline 555 & 14.955 & 0.76 & 6.14 & 0.37 & 0.023 & $\mathrm{M}$ & 1 & BJG 1608 & \\
\hline 556 & 14.956 & 0.75 & 7.19 & 0.38 & 0.018 & M & 1 & BJG 4335 & \\
\hline 602 & 15.098 & 0.81 & 7.35 & 0.44 & 0.032 & M & 1 & BJG 3935 & \\
\hline 620 & 15.172 & 0.82 & 7.41 & 0.57 & 0.026 & M & 1 & BJG 3168 & \\
\hline 633 & 15.201 & 0.81 & 7.90 & 0.80 & 0.019 & $\mathrm{M}$ & 1 & BJG 1641 & \\
\hline 649 & 15.264 & 0.84 & 8.31 & 0.57 & 0.012 & $\mathrm{M}$ & 1 & BJG 1917 & \\
\hline 652 & 15.266 & 0.79 & 7.47 & 0.58 & 0.016 & M & 1 & BJG 3964 & \\
\hline 657 & 15.279 & 0.85 & 7.59 & 0.48 & 0.036 & - & 1 & & \\
\hline 699 & 15.365 & 0.85 & 7.60 & 0.79 & 0.029 & M & 1 & BJG 2997 & \\
\hline 713 & 15.399 & 0.86 & 7.96 & 0.64 & 0.032 & M & 1 & BJG 2704 & \\
\hline 752 & 15.496 & 0.85 & 8.23 & 0.51 & 0.014 & M & 2 & BJG 3852 & PDM breaks ambiguity \\
\hline 772 & 15.552 & 0.86 & 8.75 & 0.79 & 0.011 & $\mathrm{M}$ & 2 & BJG 3597 & LS ambiguous \\
\hline 796 & 15.609 & 0.91 & 8.63 & 0.61 & 0.022 & - & 1 & & \\
\hline 807 & 15.637 & 0.99 & 8.92 & 0.63 & 0.018 & M & 1 & BJG 915 & 2 spot groups \\
\hline 862 & 15.771 & 0.96 & 8.75 & 0.72 & 0.040 & - & 1 & & \\
\hline 864 & 15.771 & 0.93 & 7.82 & 0.54 & 0.065 & - & 1 & & \\
\hline 872 & 15.782 & 0.96 & 7.52 & 0.45 & 0.049 & - & 1 & & \\
\hline 898 & 15.826 & 0.90 & 7.36 & 0.54 & 0.036 & M & 1 & BJG 1828 & \\
\hline 909 & 15.838 & 0.97 & 8.03 & 0.55 & 0.019 & - & 1 & & \\
\hline 920 & 15.866 & 0.94 & 8.65 & 0.67 & 0.017 & - & 1 & & \\
\hline 921 & 15.868 & 0.98 & 7.86 & 0.47 & 0.022 & - & 1 & & \\
\hline 923 & 15.869 & 0.95 & 8.87 & 0.69 & 0.022 & - & 1 & & \\
\hline 931 & 15.889 & 0.98 & 8.75 & 0.68 & 0.025 & - & 1 & & \\
\hline 935 & 15.897 & 0.96 & 9.24 & 0.75 & 0.037 & - & 1 & & \\
\hline 937 & 15.902 & 0.96 & 7.52 & 0.50 & 0.035 & M & 1 & BJG 2546 & \\
\hline 954 & 15.922 & 0.97 & 9.70 & 0.79 & 0.013 & M & 2 & BJG 1703 & Low Amplitude \\
\hline 969 & 15.956 & 0.98 & 9.55 & 0.90 & 0.021 & $\mathrm{~N}$ & 1 & BJG 2435 & \\
\hline 974 & 15.966 & 0.98 & 9.27 & 0.68 & 0.016 & M & 1 & BJG 2033 & \\
\hline 975 & 15.967 & 1.00 & 9.58 & 0.62 & 0.028 & M & 1 & BJG 578 & \\
\hline 1227 & 16.409 & 1.08 & 8.83 & 0.72 & 0.064 & M & 1 & BJG 1362 & \\
\hline 1455 & 16.730 & 1.17 & 4.81 & 0.24 & 0.021 & M & 2 & BJG 2505 & Noisy, PDM picks $\sim 9.6 \mathrm{~d}$ \\
\hline 1456 & 16.730 & 1.13 & 13.31 & 1.58 & 0.026 & $\mathrm{M}$ & 1 & BJG 4598 & \\
\hline 1605 & 16.946 & 1.22 & 5.81 & 0.30 & 0.083 & M & 1 & BJG 4556 & \\
\hline 1711 & 17.091 & 1.22 & 10.22 & 0.84 & 0.063 & M & 1 & BJG 3794 & \\
\hline 1744 & 17.130 & 1.23 & 6.79 & 0.44 & 0.026 & - & 2 & & Noisy, PDM picks $\sim 13.3 \mathrm{~d}$ \\
\hline 2010 & 17.483 & 1.25 & 5.82 & 0.26 & 0.056 & - & 1 & & \\
\hline 2071 & 17.568 & 1.35 & 7.30 & 0.57 & 0.088 & M & 1 & BJG 2439 & \\
\hline 2285 & 17.805 & 1.35 & 3.26 & 0.10 & 0.075 & $\mathrm{~N}$ & 1 & BJG 2458 & \\
\hline 2346 & 17.852 & 1.46 & 9.68 & 0.72 & 0.073 & M & 1 & BJG 771 & \\
\hline 2632 & 18.161 & 1.55 & 1.67 & 0.03 & 0.117 & M & 1 & BJG 3428 & \\
\hline
\end{tabular}

Notes. Mem = M, N, - respectively indicate BJG05 members, non-members, and stars without BJG05 membership information. $Q=1,2$ respectively indicate high quality and lower quality periods. 


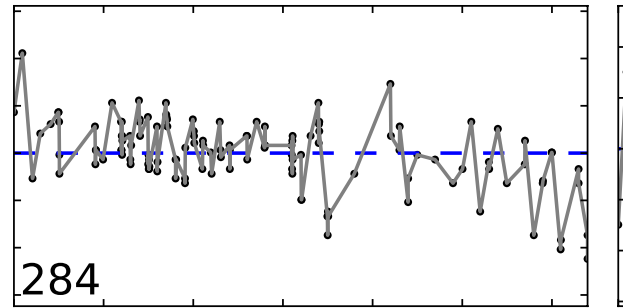

-

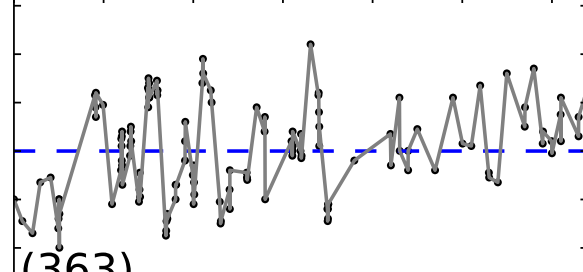
(363)

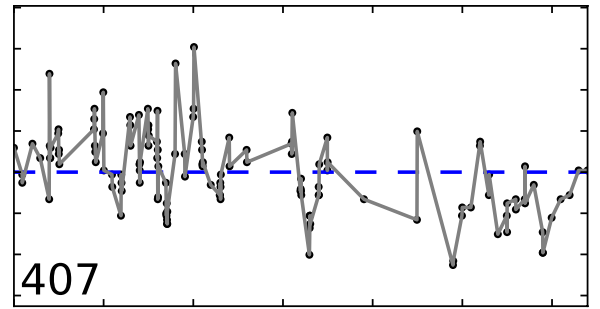

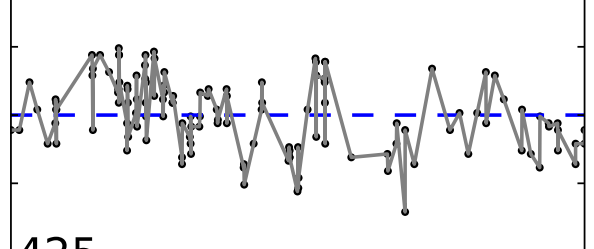
$-425$
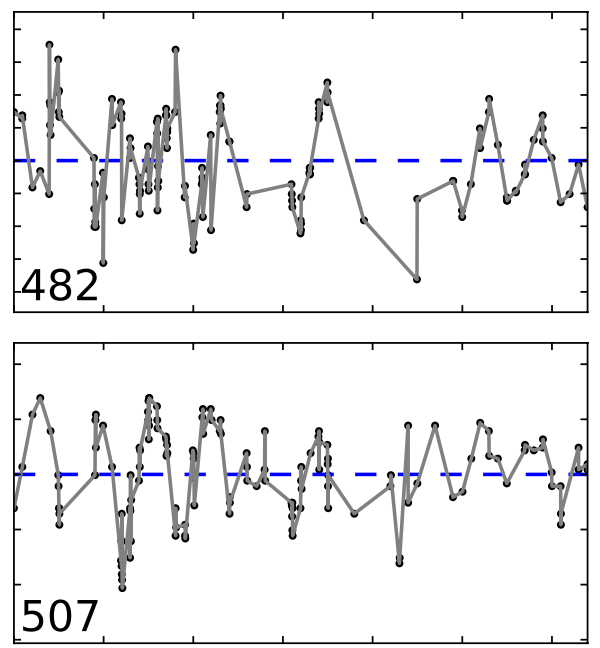
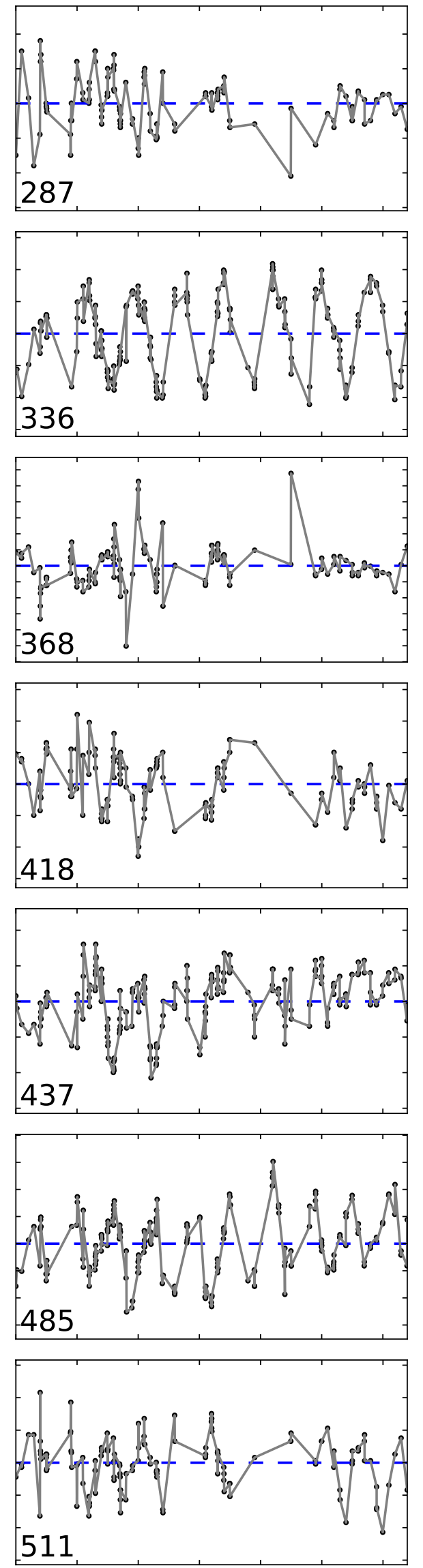
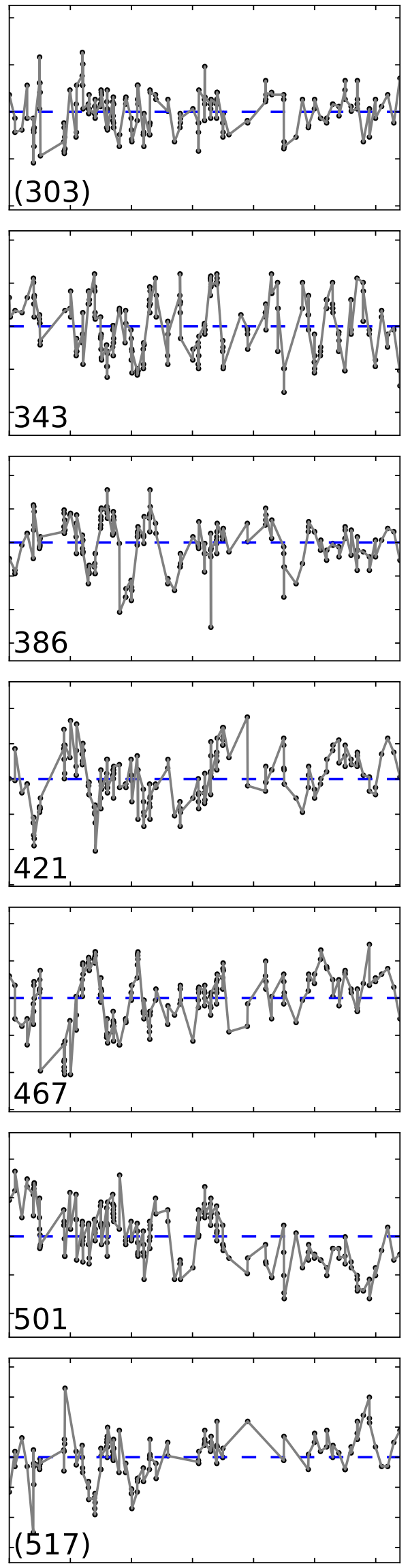

Fig. B.1. Light curves 1 ( $Q=2$ periods in parentheses, $x$-units $=10 \mathrm{~d}, y$-units $=0.01 \mathrm{mag}$ ). 


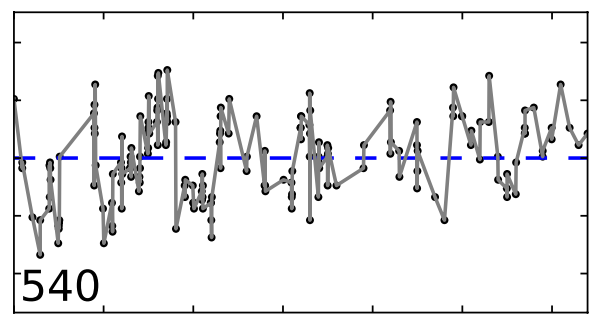

(602.

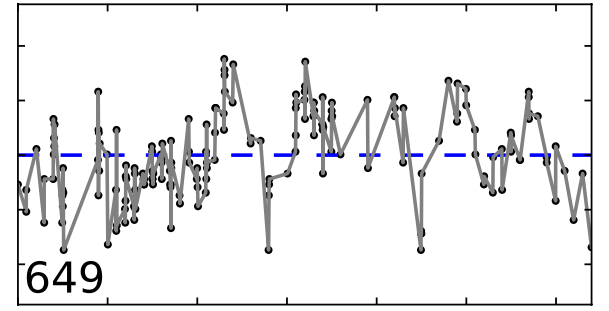

(699.)

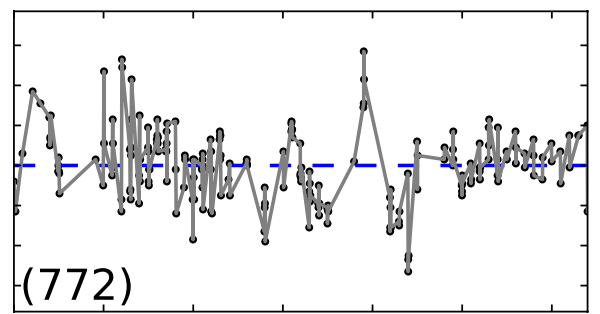

862 .

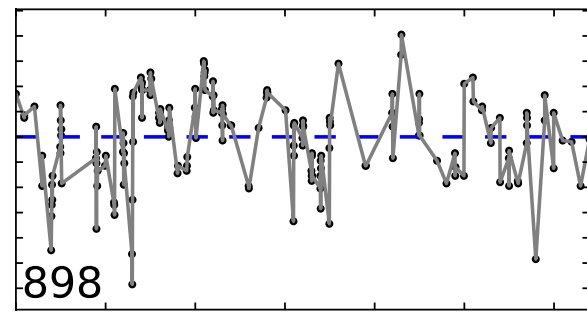

\section{2}
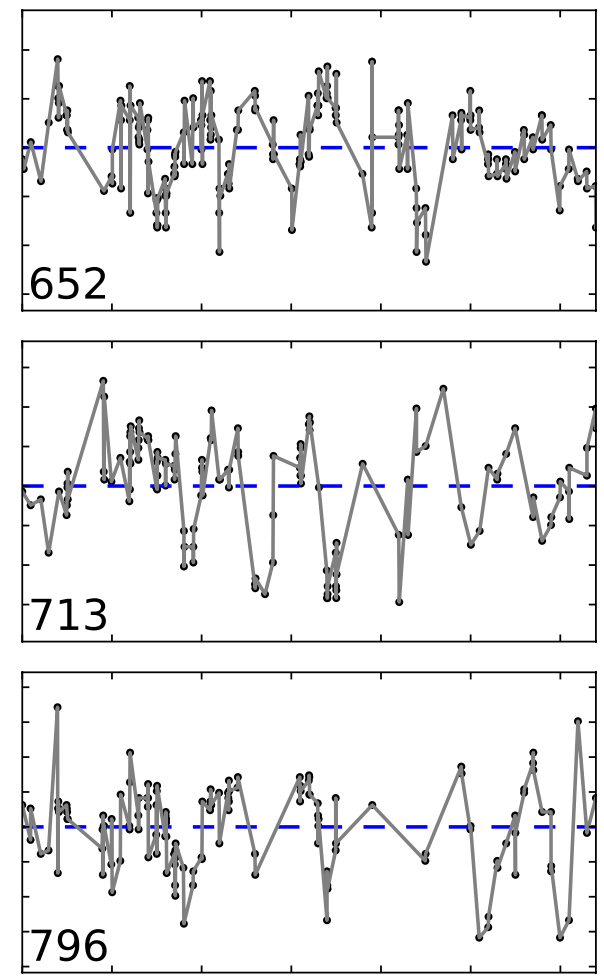

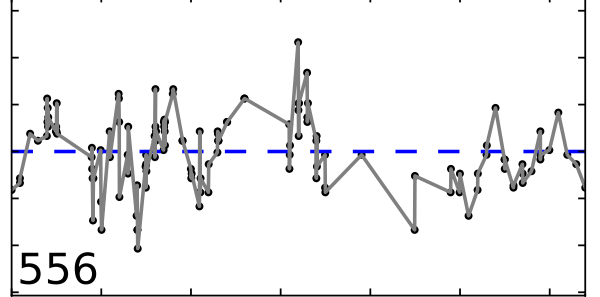

-

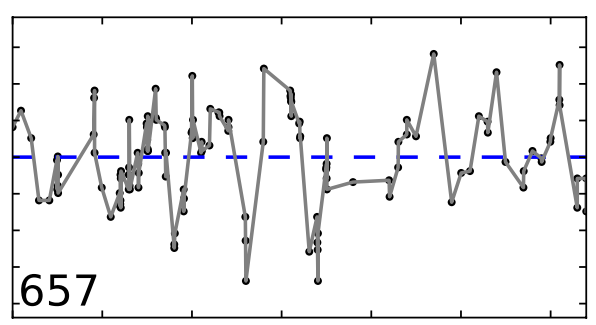

657
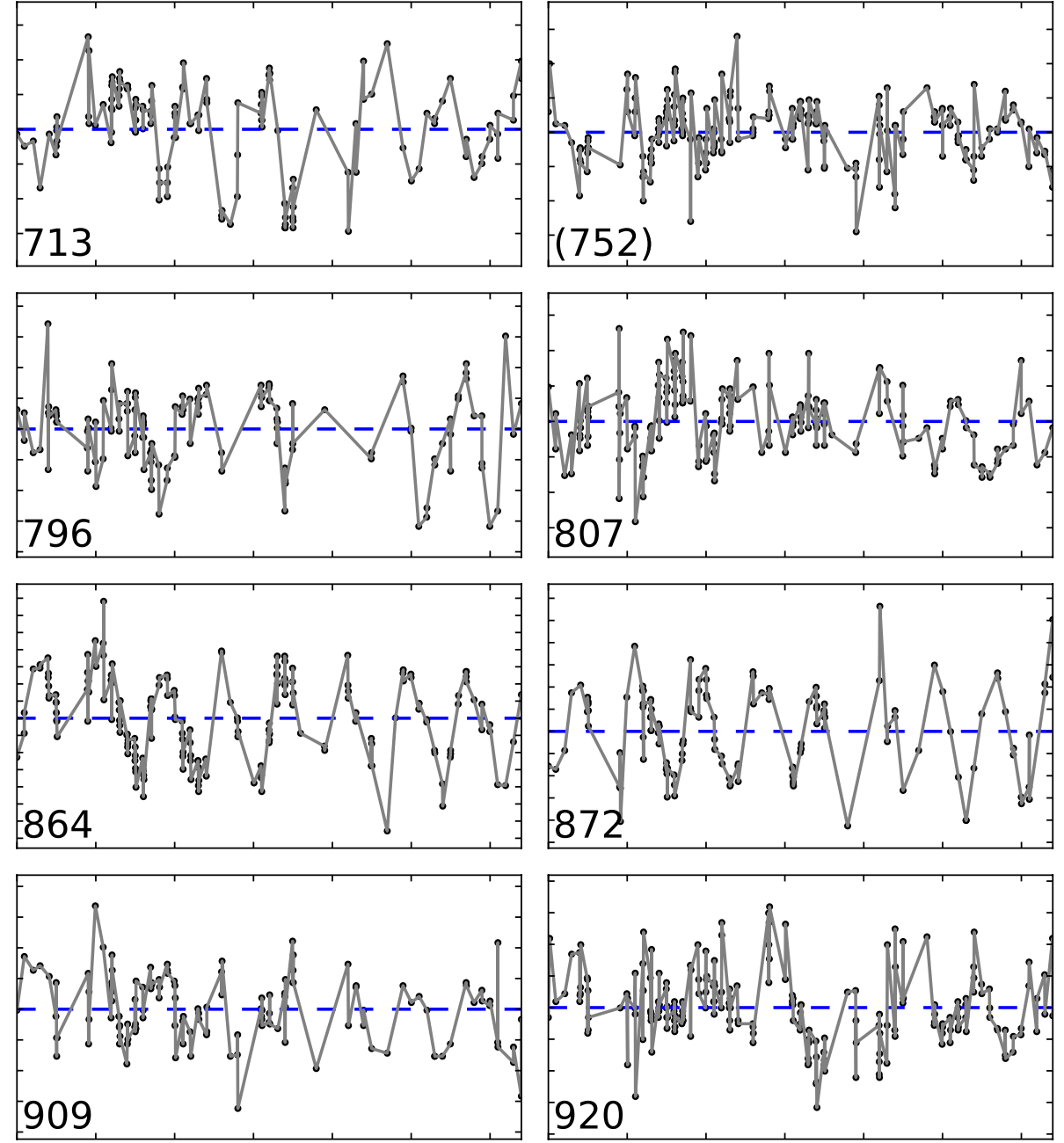

Fig. B.2. Light curves $2(Q=2$ periods in parentheses, $x$-units $=10 \mathrm{~d}, y$-units $=0.01 \mathrm{mag}$ ). 

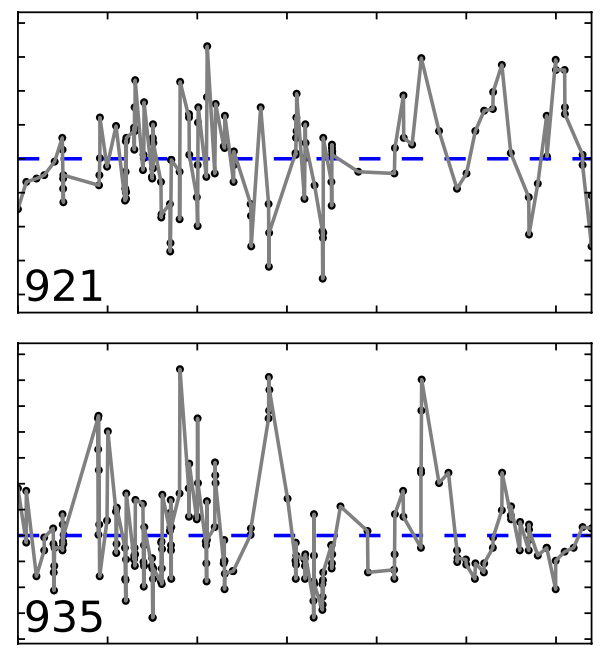
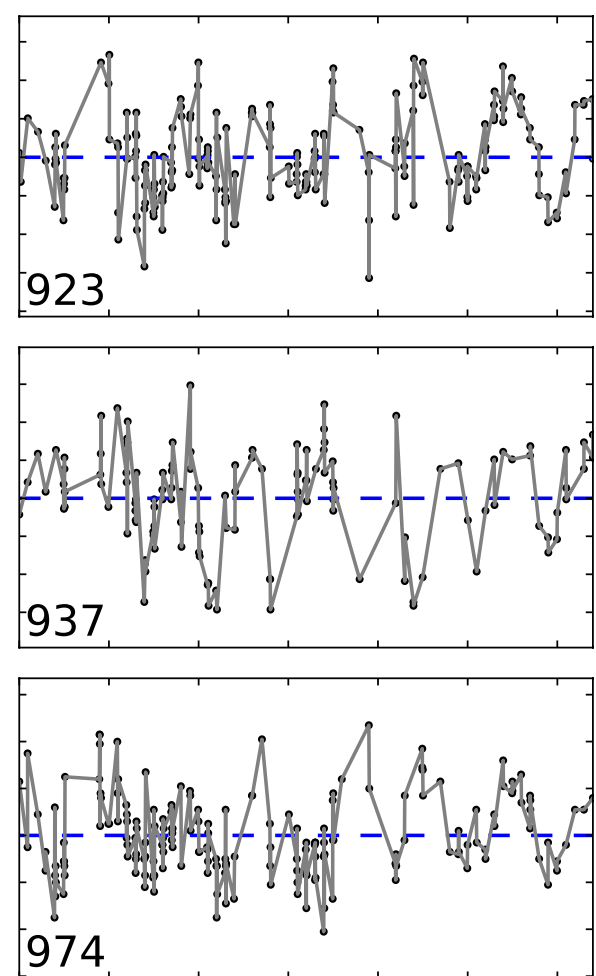
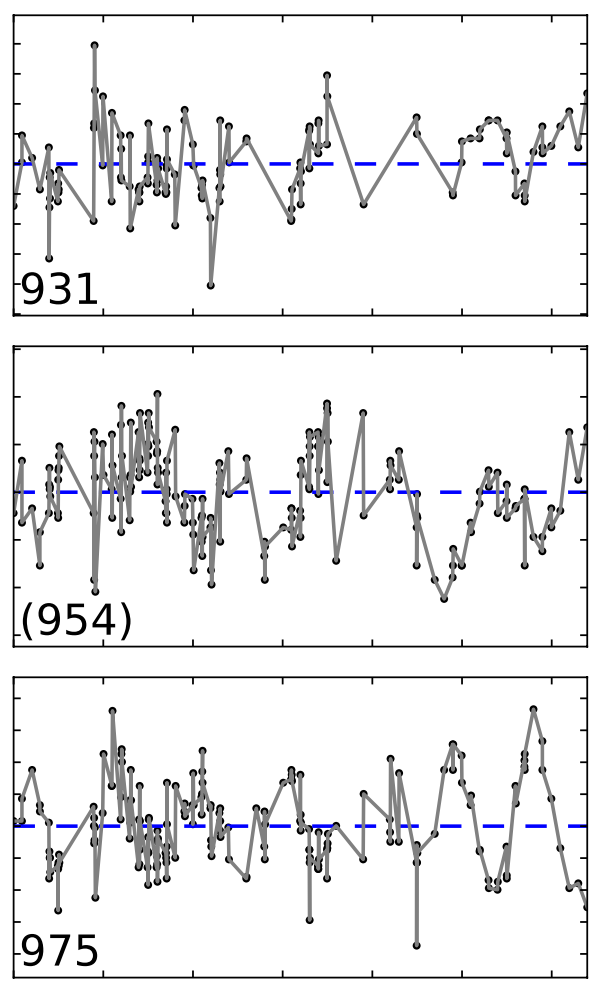

$-975$
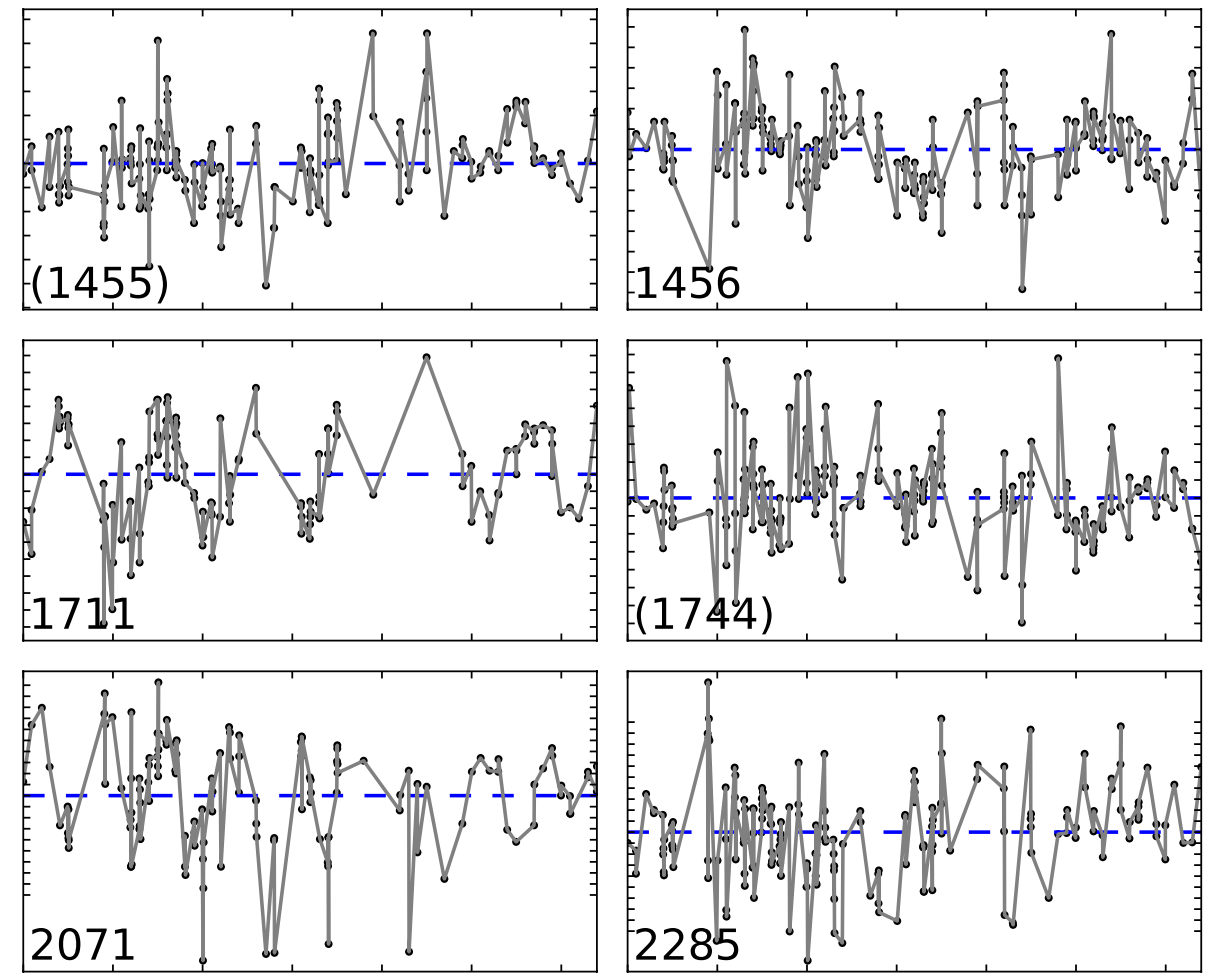

(1744)
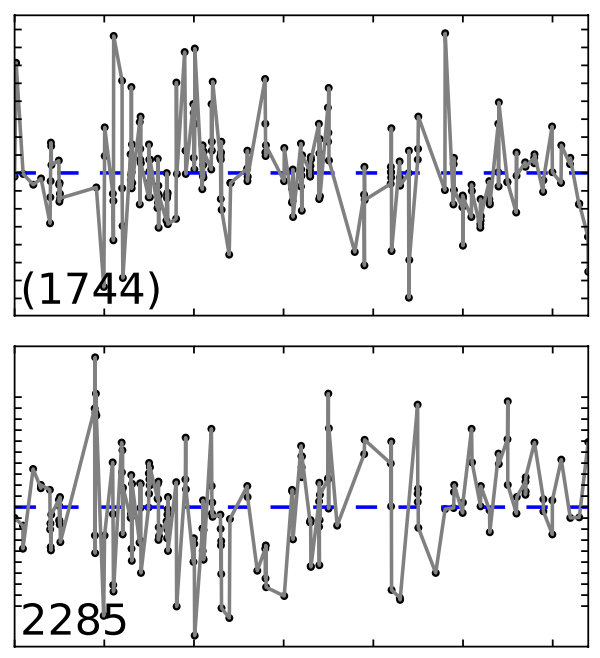
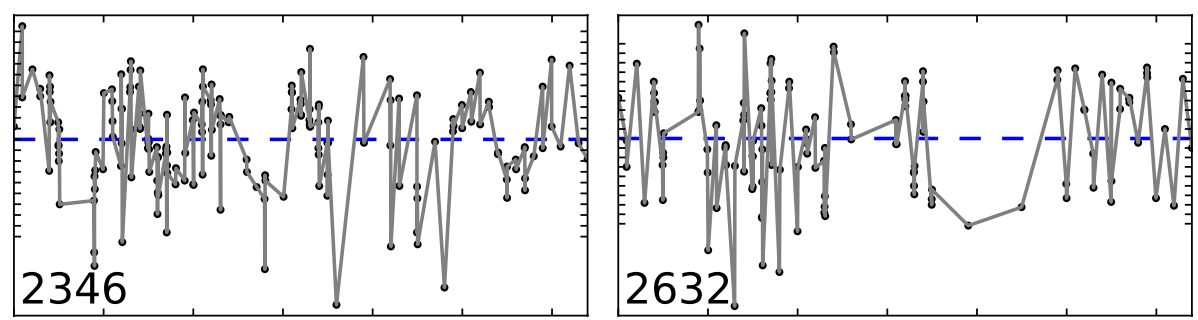

Fig. B.3. Light curves 3 ( $Q=2$ periods in parentheses, $x$-units $=10 \mathrm{~d}, y$-units $=0.01 \mathrm{mag}$ ). 

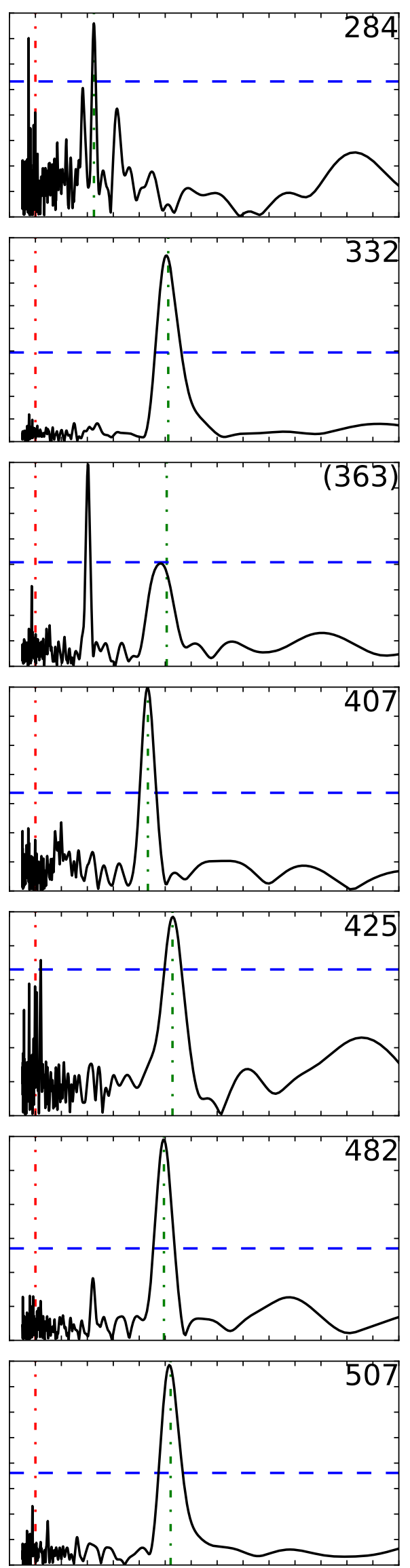
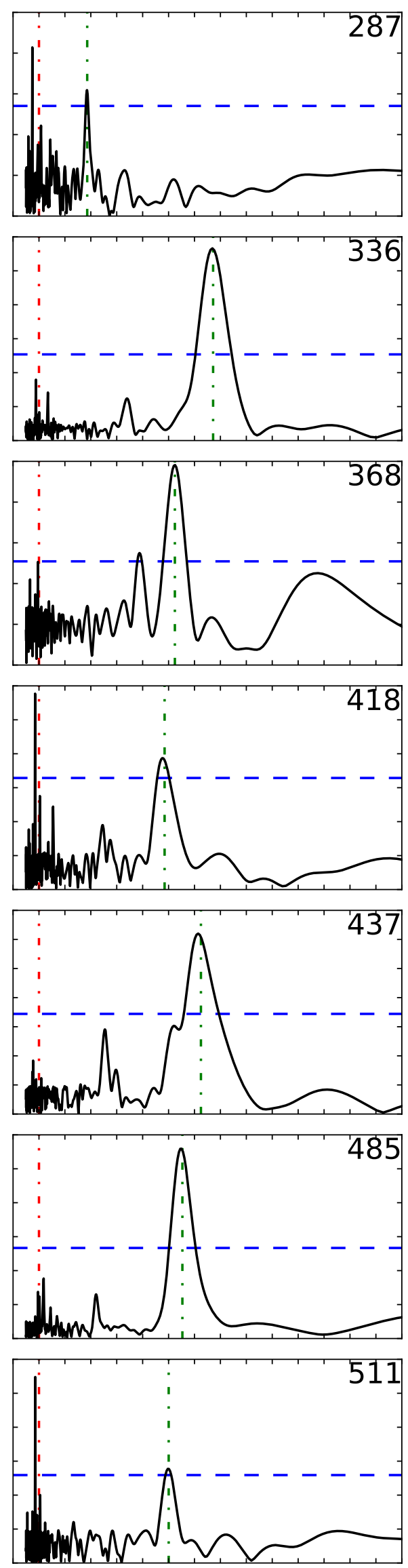
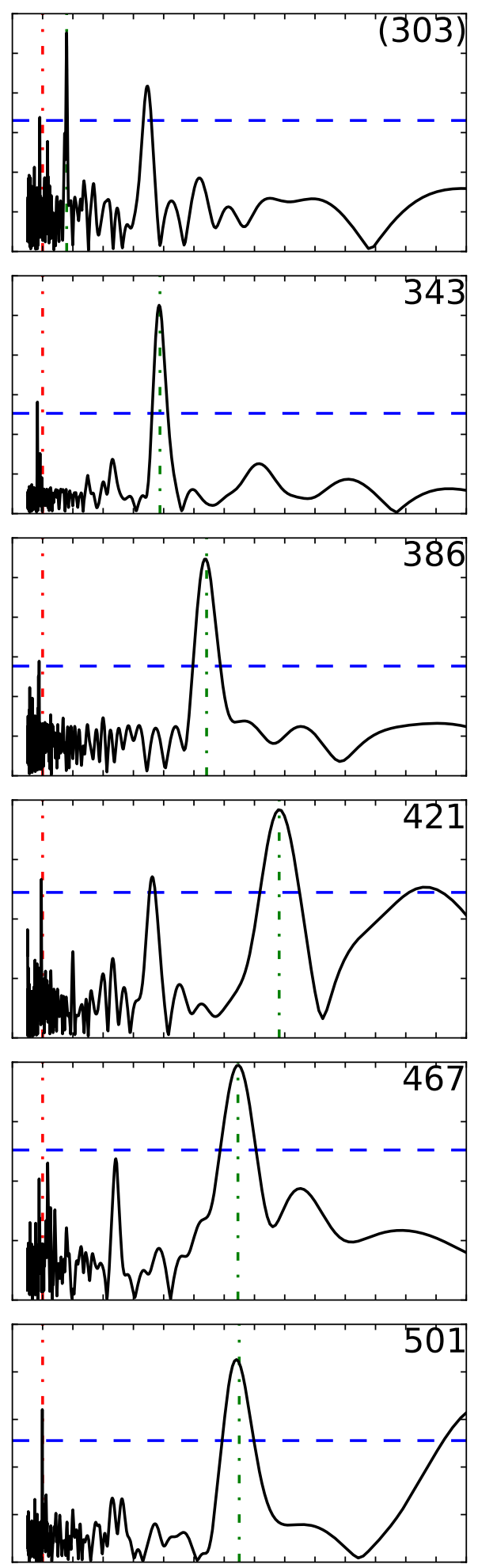

(517)

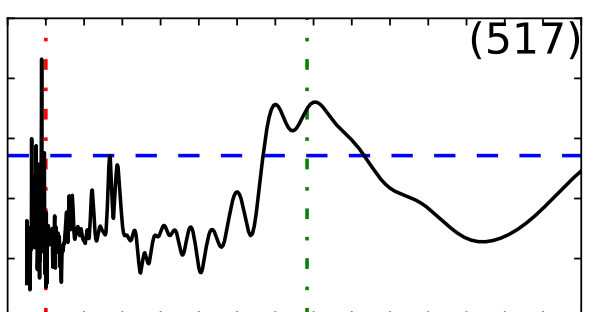

Fig. B.4. Spectra 1 ( $Q=2$ periods in parentheses, $x$-units $=1 \mathrm{~d}, y$-units arbitrary, selected rotation period marked with green line, $5 \sigma$ level marked with blue line. 
S. A. Barnes et al.: Color-period diagram for M 48
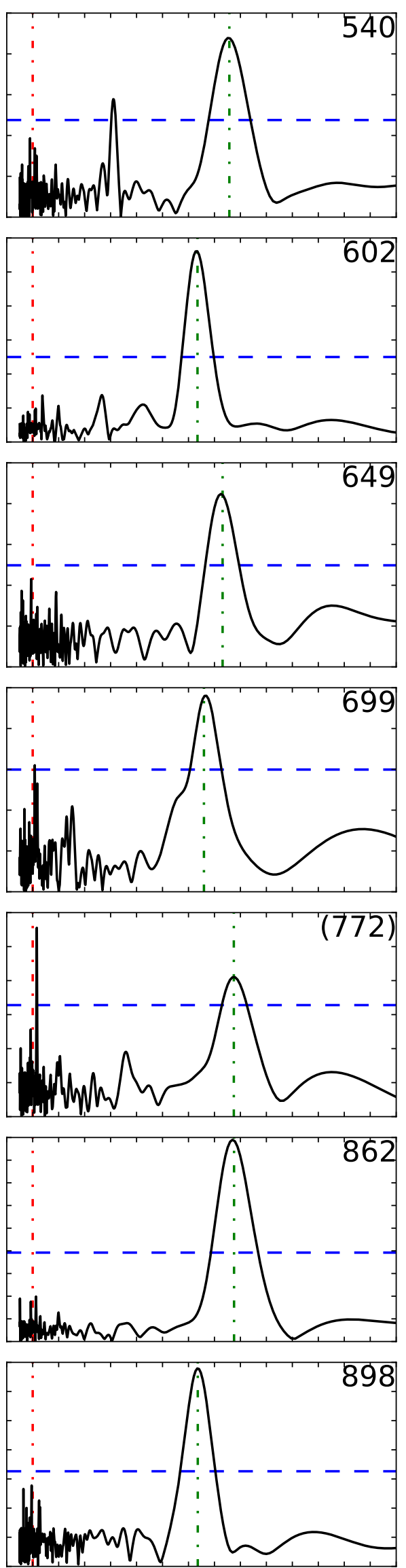

Fig. B.5. Spectra $2(Q=2$ periods in parentheses, $x$-units $=1 \mathrm{~d}$, $y$-units arbitrary, selected rotation period marked with green line, $5 \sigma$ level marked with blue line).
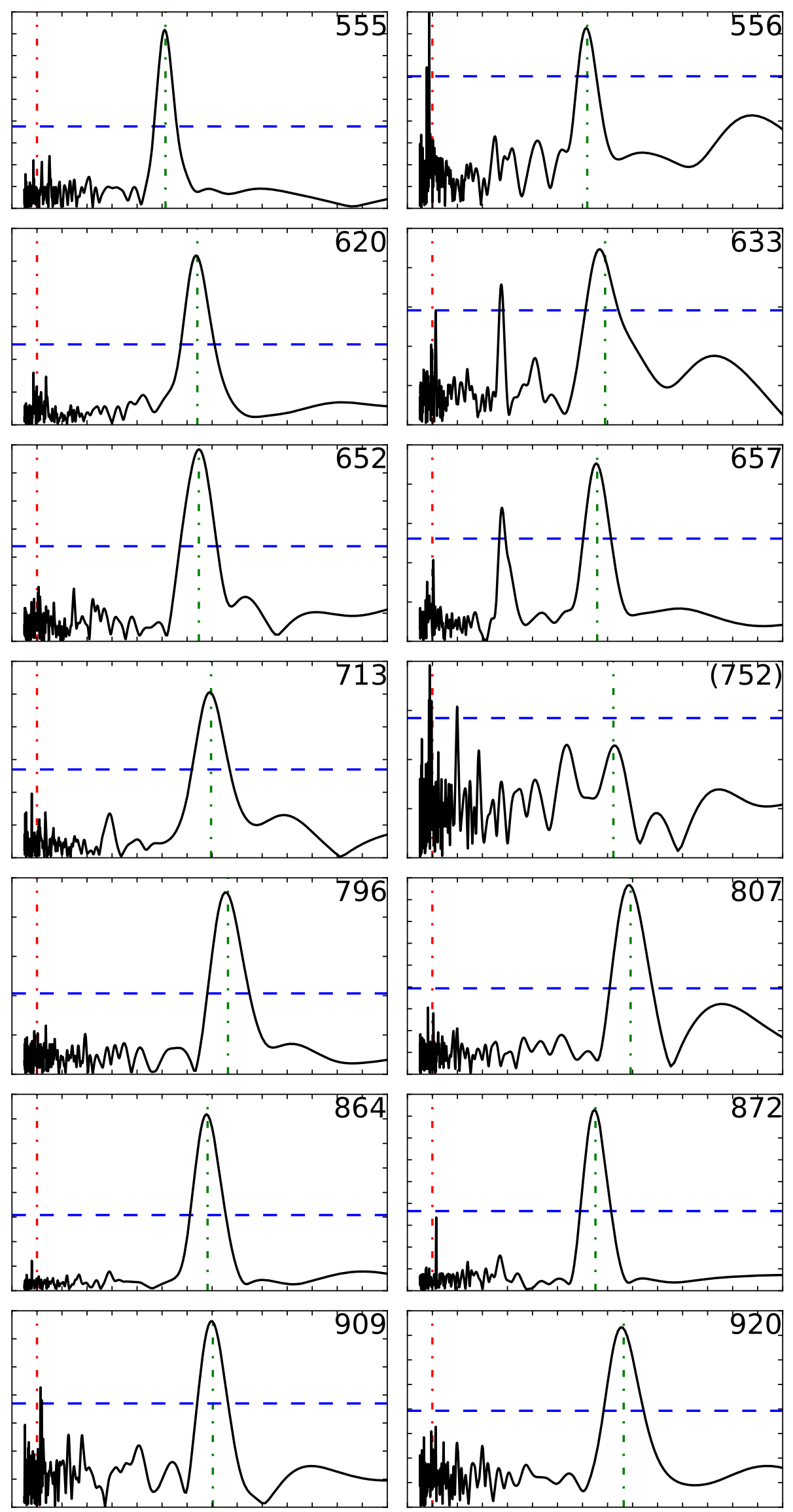

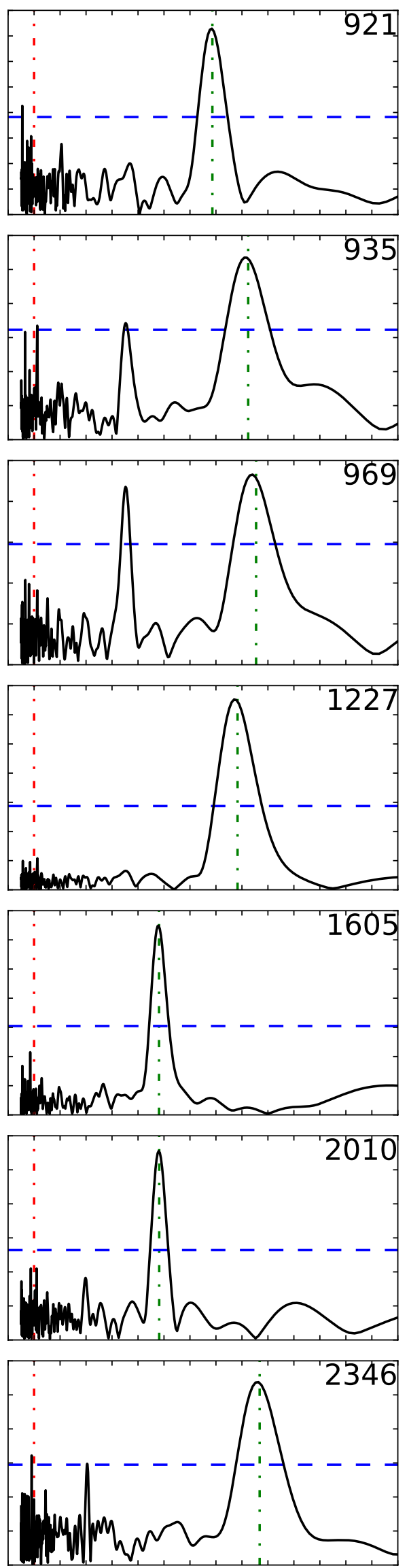

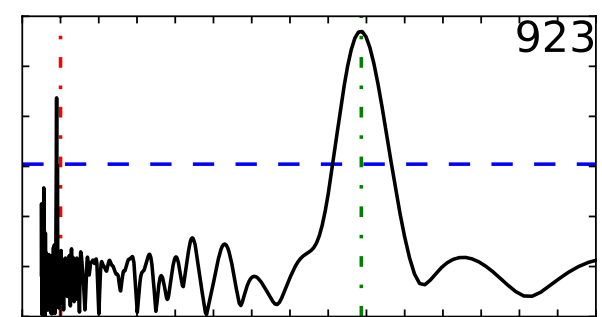

937
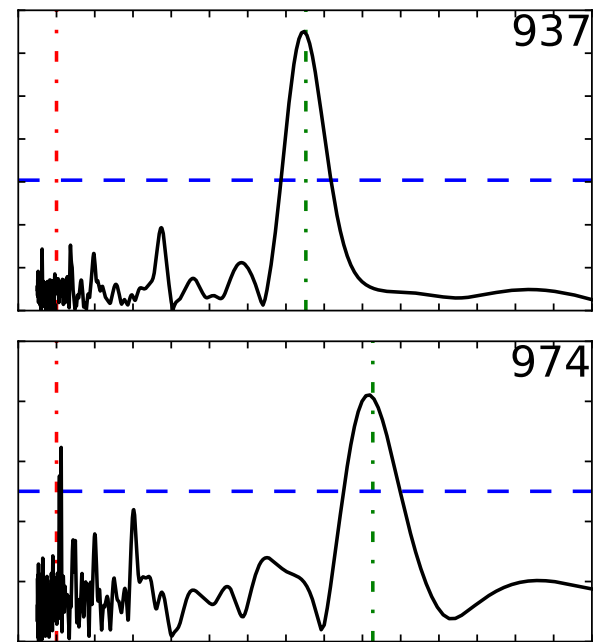

(1455)
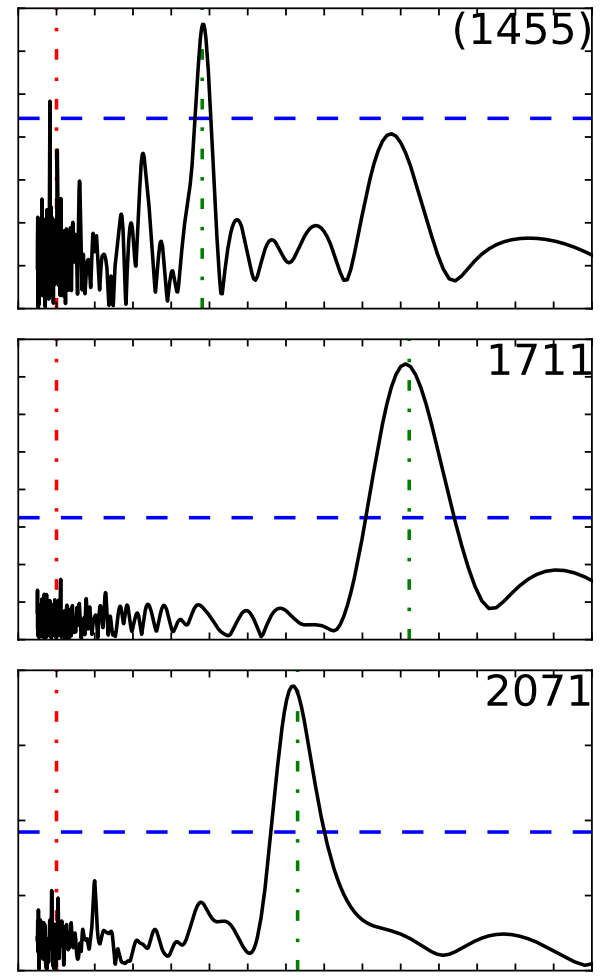

2632

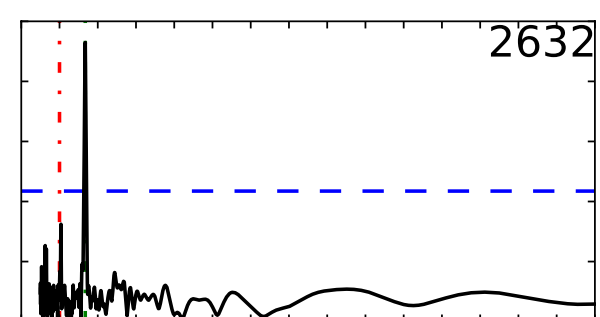

Fig. B.6. Spectra 3 ( $Q=2$ periods in parentheses, $x$-units $=1 \mathrm{~d}, y$-units arbitrary, selected rotation period marked with green line, $5 \sigma$ level marked with blue line). 

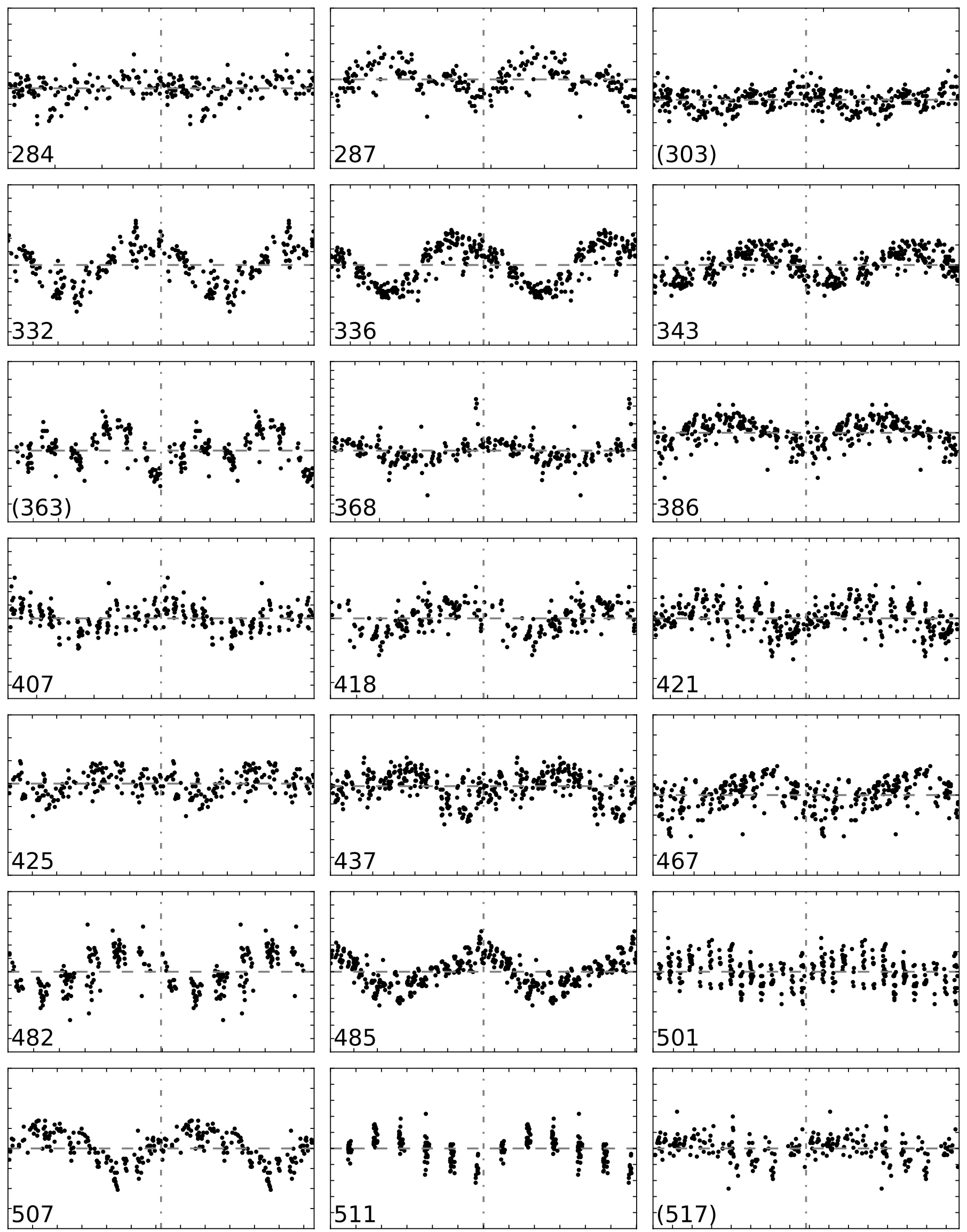

Fig. B.7. Phased curves $1(Q=2$ periods in parentheses, $x$-units $=1 \mathrm{~d}, y$-units $=0.01 \mathrm{mag}$ ), with two phases displayed for each star. 

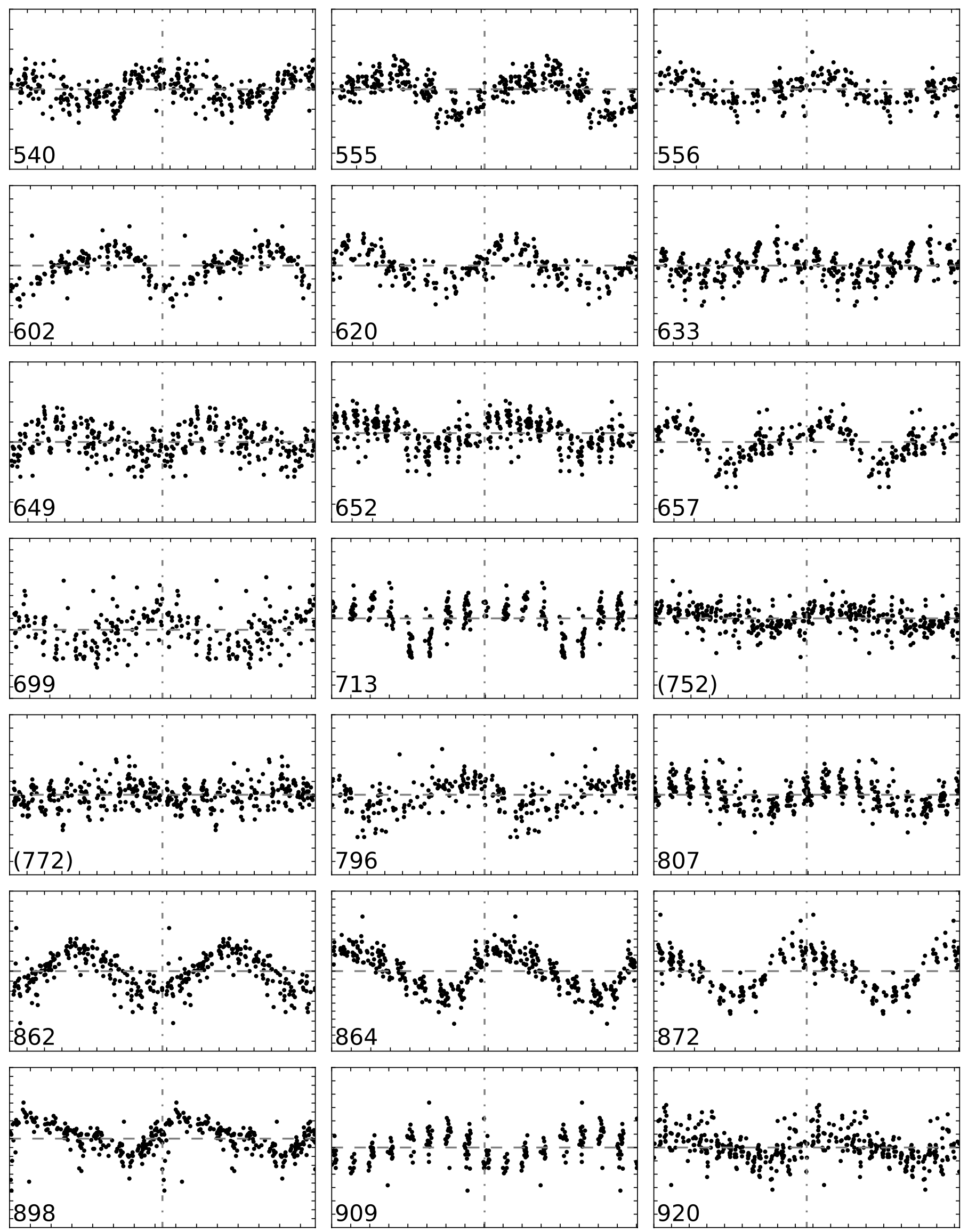

Fig. B.8. Phased curves $2(Q=2$ periods in parentheses, $x$-units $=1 \mathrm{~d}, y$-units $=0.01 \mathrm{mag}$ ), with two phases displayed for each star. 

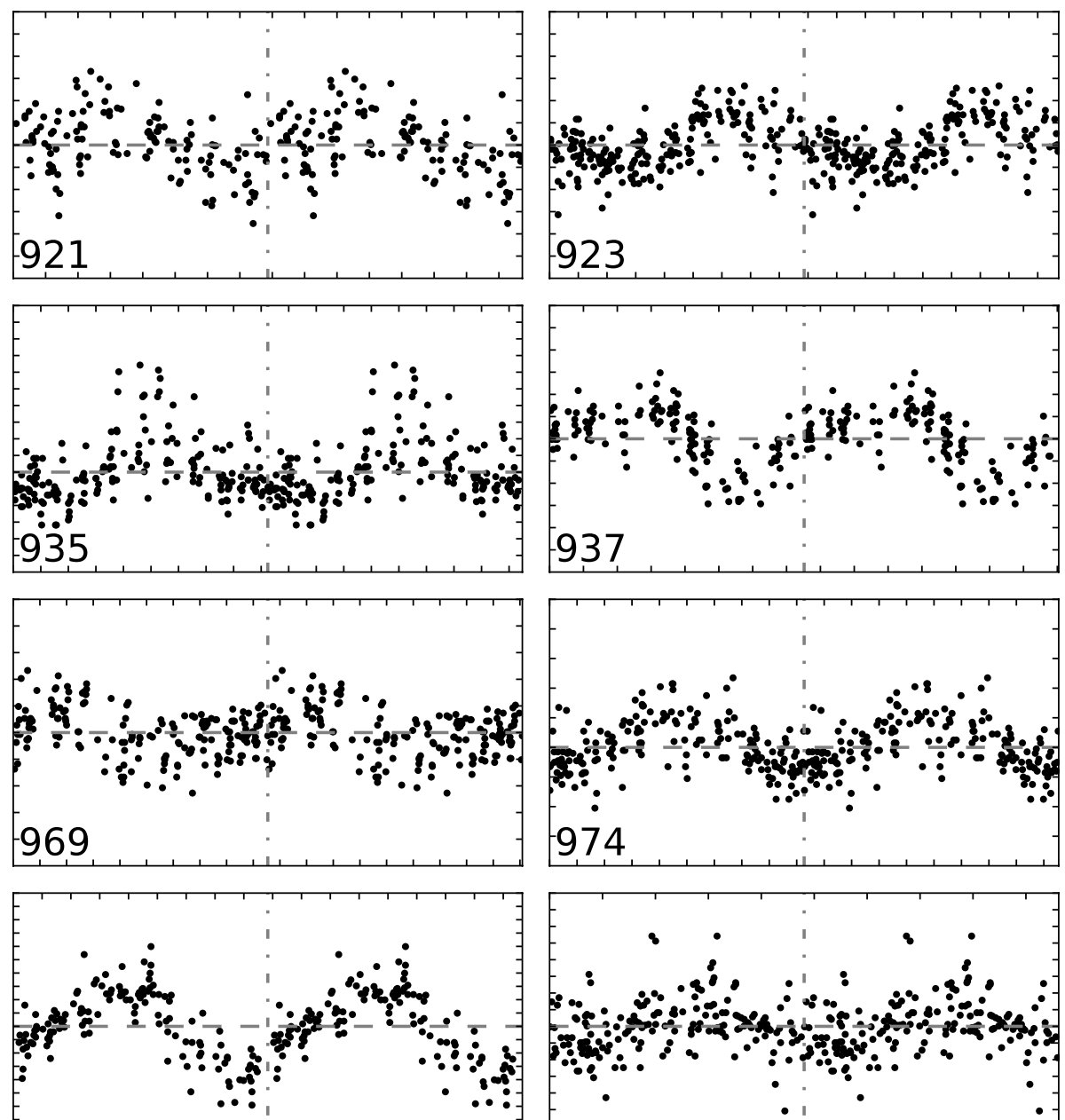

1227
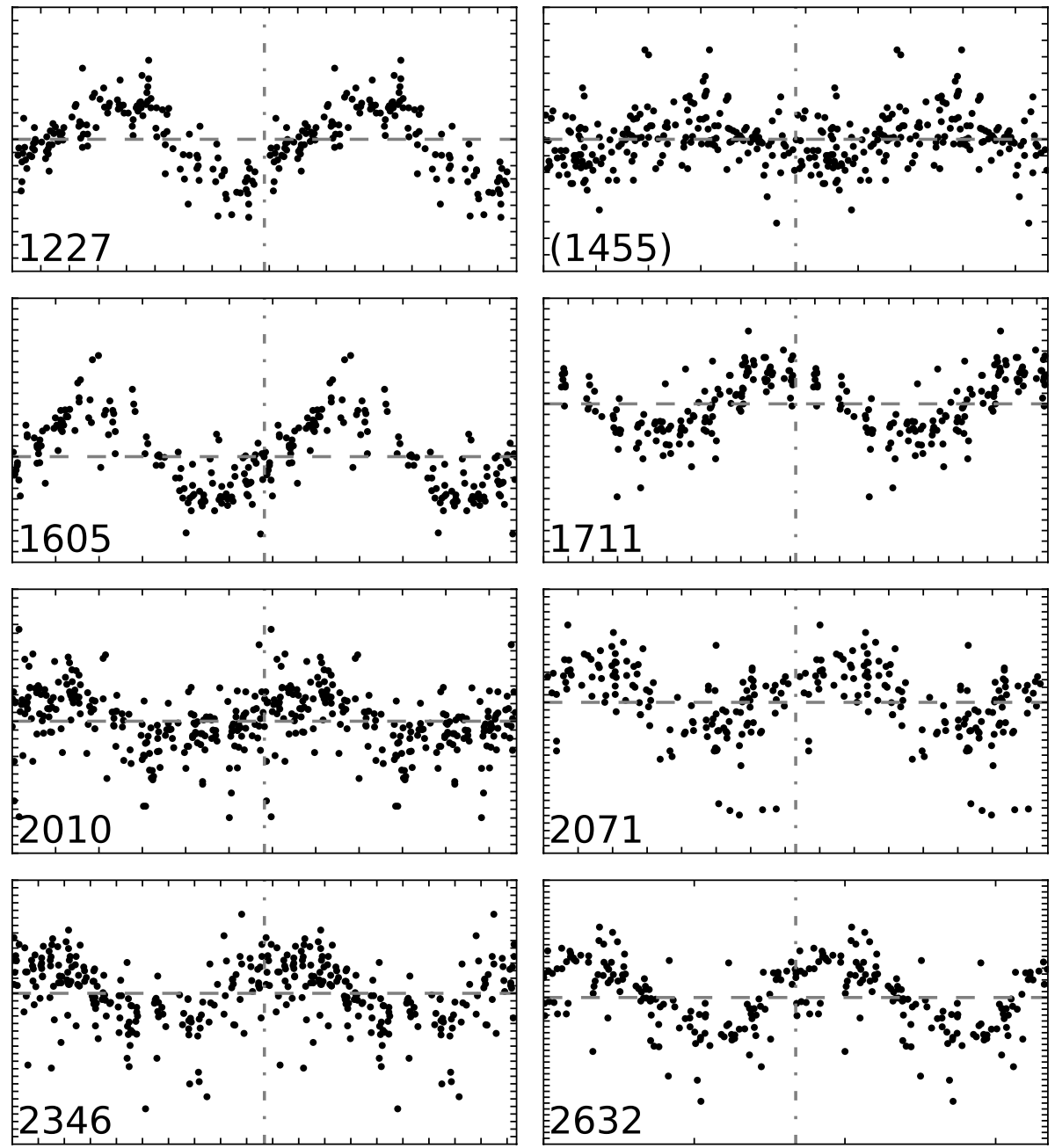
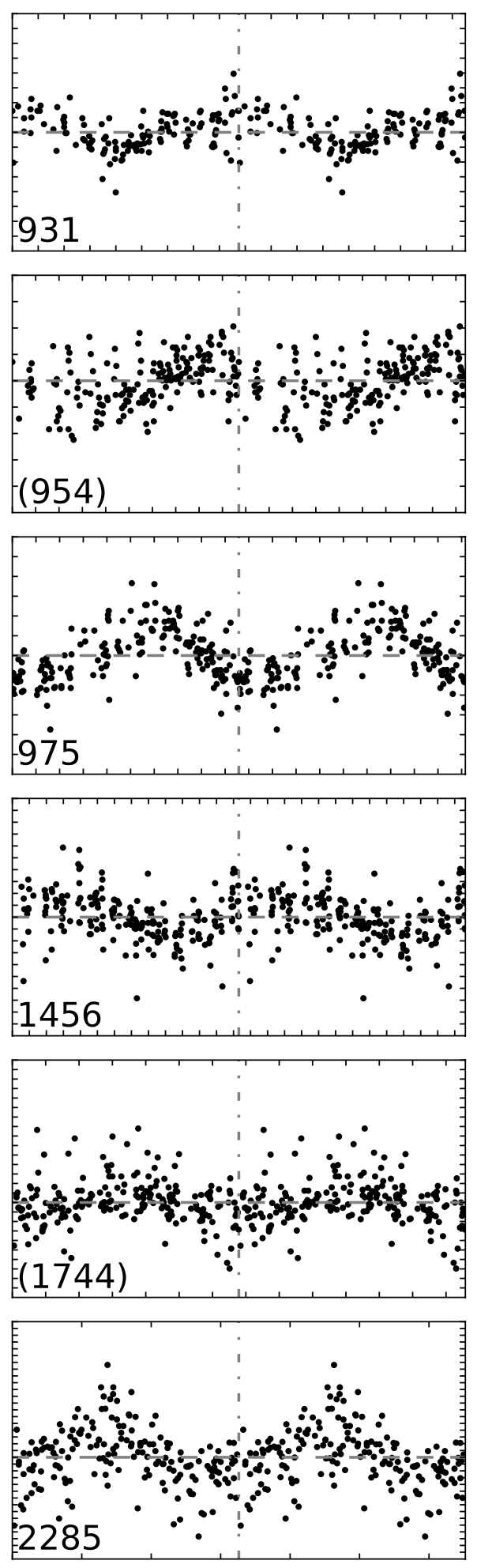

Fig. B.9. Phased curves 3 ( $Q=2$ periods in parentheses, $x$-units $=1 \mathrm{~d}$, $y$-units $=0.01 \mathrm{mag}$ ), with two phases displayed for each star. 